\title{
Studien über den Einfluss der Einatmung von Sauerstoff, sowie von kohlensäurereicher bzw. sauerstoffarmer Luft auf den Energieumsatz und den intermediären Kohlehydratstoffwechsel.
}

\author{
I. Mitteilung. \\ Beeinflussung des Milchsäurestoffwechsels. \\ Von \\ Kongo Kodera. \\ (古寺: 金 㓮) \\ (Aus der Medizinischen Klinik von Prof. Dr. T. Kato, \\ an der Kaiscrlichen Universität zu Scndai.)
}

\section{Einleitung.}

Es ist eine allgemein ancrkannte Tatsache, dass der Prozess, wobei die bei Muskelkontraktion durch eine Abspaltung aus Glykogen entstandene Milchsäure wiederum zu Glykogen resynthetisiert wird, durch die Blutzirkulation, Sauerstoffversorgung und Wassertoffionenkonzentration in der Muskulatur beeinflusst werden kann. Und derartige Faktoren sind auch ihrerseits durch ihre jeweilige Veränderungen gegenseitig Schwankungen unterworfen. Sie werden aber auch durch die Einatmung der kohlensäurereichen bzw. sauerstoffarmen Luft beeinflusst, indem die Einatmung derselben die Tiefe und Frequenz der Lungenatmung zur Veränderung bringt und auch öfters zu behinderter oder beschleunigter Abgabe der Kohlensäure führt, wodurch der in den Alveolen herrschende $\mathrm{CO}_{2}$-Partialdruck und folglich auch der Gehalt an $\mathrm{H}_{2} \mathrm{CO}_{3}$ im Serum Veränderungen erfahren.

Da aber die Wasserstoffionenkonzentration $(\mathrm{cH})$ des Blutes durch die Grösse des Verhältnisses von $\mathrm{H}_{2} \mathrm{CO}_{3}$ zu NaHCO 3 im Blute bestimmt wird, muss die durch die Einatmung von kohlensäurereicher bzw. sanerstoffarmer Luft bedingte Änderung der Lungenatmung naturgemäss auch die Änderung der $\mathrm{cH}$ des Blutes mit sich bringen.

In bezug darauf sind in der Literatur unzählbare Arbeiten niedergelegt worden. 
Über den Einfluss der Einatmung der kohlensäurereichen Luft ist von Haldane u. Priestley, ${ }^{13}$ Hill u. Flack, ${ }^{3}$ F.H.Scott, ${ }^{3}$ Hough, ${ }^{4}$ Laqueur u. Verzár, ${ }^{5}$ Campbell, Douglas, Haldane u. Hobson, ${ }^{(i)}$ Campbell, Douglas u. Hobson, , Hoocker, Wilson u. Connett, ${ }^{8}$ R. W. Scott, ${ }^{9}$ Henderson u. Haggard, ${ }^{10}$ Collip, ${ }^{11)}$ Douglas u. Haldane, $\left.{ }^{19}\right)$ Dale u. Evans, ${ }^{13)}$ Schneider, ${ }^{14}$ Schneider . Truesdell, ${ }^{15}$ Sasaki, ${ }^{16}$ Sppinger, Kisch u. Schwarz, ${ }^{17}$ Gollwitzer-Meier, ${ }^{18}$ ) Grüneberg u. Viethen, ${ }^{19}$ Hayasaka u. Itakura, ${ }^{20}$ Herxheimer u. Kost ${ }^{21}$ übereinstimmend angegeben worden, dass wenn der $\mathrm{CO}_{2}$-Gehalt der inspirierten Luft 5-6\% erreicht hat, die $\mathrm{H}_{2} \mathrm{CO}_{3}$ des Blutes merklich zunähme und durch diese Zunahme das Atemzentrum gereizt werde, was zur Ventilationssteigerung führe.

Laubende $\mathrm{r}^{22}$ ) hat auch bei Kaninchen nachgewiesen, dass durch die Einatmung von $6-8 \% \mathrm{CO}_{2}$ das $p \mathrm{H}$ des Blutes um $0,2-0,3$ sinkt, $\mathrm{Sch}$ wieg $\mathrm{k}^{23}$ hat auch festgestellt, dass durch die Einatmung von $8 \% \mathrm{CO}_{2}$ das Blut- $p \mathrm{H}$ um 0,12 0,18 nach saurer Seite verschoben wird, auch Eppinger u. a. machten die Beobachtung, dass durch die Einatmung von $10 \% \mathrm{CO}_{2}$ das $p \mathrm{H}$ des Blutes von 7,46 bis unter 7,32 absinkt.

Es existieren über die Veränderung der Atmung bei der Inhalation sauerstoffarmer Luft die Arbeiten von vielen Forschern, wie Schumburg u. Zuntz, ${ }^{24)}$ Jaquet u. Stähelin, ${ }^{25}$ Haldane u. Paulton, ${ }^{26)}$ Barcroft, Ca-

\footnotetext{
1) Haldane u. Priestley, Journ. Physiol, 1905, 32, 225.

2) Hill n. Flack, Journ. Physiol, 1908, 37, 77.

3) F. H. S cott, Joul'n. Physiol., 1908, 37, 301.

4) Hough, Americ. Journ. Physiol., 1911, 28, 369.

5) Laqueur u. Verzár, Pflüger's Arch., 1912, 143, 395.

6) Campbell, Donglas, Haldane u. Hobson, Journ. Physiol., 1913, 46, 301.

7) Campbell, Douglas u. Hobson, Journ. Physiol, 1914, 48, 303.

8) Hoooker, Wilson u. Connett, Americ. Joun. Physiol., 1917, 43, 351.

9) R. W. Stott, Americ. Journ. Physiol., 1917, 44, 196.

10) Henderson u. Haggard, Journ. biol. Chem., 1918, 33, 333.

11) Collip. Journ. Physiol, 1920, 54, 58.

12) Douglasu. Haldane, Journ. Plysiol., 1922, 56, 69.

13) Dale u. Evans, Journ. Physiol., 1922, 56, 125.

14) Sehneider, Amerie. Jonu. Physiol, 1922, 59, 449.

15) Schneider u. Truesde11, Americ. Journ. Physiol., 1922, 63, 155.

16) Sasaki, Journ. Biophysics, 1927, 2, 197.

17) Eppinger, hisch $\mathbf{h}$. Sehwarz, Das Versagen des Kreislaufes, Berlin, 1927,

18) Gollwitzex-Meier, Pflüg gor's Arch., 1929, 222, 104.

19) Grü neberg n. Viethen, Jo. kinderheilk, 1930, 128, 65, ref. in 1931, 60,

20) Hayasaka n. Itakura, Tohoku Journ. Exp. Med., 1931, 18, 166.

21) Herxheimer u. Kost, Zeitschr. kl. Med., 1931, 116, 88.

22) La ubender, Sehmiedeberg's Al'ch., 1932, 165, 5.

23) Schwie gk, Zeitschr. f. ges. exp. Med., 1930, 74, 274.

24) Schumbul'g u. Zuntz, P f litger's Arch., $1896,63,461$.

25) Jaquet u. Stähelin, Schm iedeberg's Arch, 1901, 46, 274.

26) Haldane u. Poulton, Journ. Physiol., 1908, 37, 390.
} 202. 324 . 
mis, Mathison, Roberts u. Ryffel,,$^{27}$ Lutz u. Schneider, ${ }^{28}$ Haldane, ${ }^{29}$ ) Haggard u. Henderson, ${ }^{30)} \mathrm{Macleod},{ }^{31)}$ Loewy, $\left.{ }^{32}\right)$ Kenster, Peemöller u. Schado $w^{33}$ sowie Yosomiya, ${ }^{34)} \mathrm{Okada}^{35}$ in hiesiger Klinik.

Nach Untersuchungen obiger Autoren nimmt die Atmungsgrösse dann erheblich $\mathrm{zu}$, wenn der $\mathrm{O}_{2}$-Gehalt in der Luft unter $14 \%$ herabgesetzt ist. Auch das Höhenklima soll die Atmung beeinflussen, so kann nämlich bei der $1200 \mathrm{~m}$ Höhe schon eine Vermehrung der Atmungsgrösse beobachtet werden und in der $2000 \mathrm{~m}$ Höhe soll eine auffallende Ventilationssteigerung zutage treten. Eine derartige Ventilationssteigerung bedingt also eine vermehrte $\mathrm{CO}_{\mathbf{2}}-\mathrm{Abgabe}$ und dementsprechend ein Absinken der cH.

Nach Angaben von $\mathrm{Henders}$ on $\mathrm{u}$. $\mathrm{Haggar} \mathrm{d}^{30}$ ) tritt beim $\mathrm{O}_{2}$-Mangel zuerst eine Hyperventilation ein, worauf das Blut der Alkalosis anheimfällt. Es hat sich hierbei herausgestellt, dass bei Zufuhr der Milchsäure zu diesem alkalotischen Blut die Azidosis nicht in demselben Masse, als sie den zugeführten Säuremenge entsprechen würde, auftritt. Nach Beobachtungen von Gesell und $H$ ertzm an $n^{36}$ ) wird bei der Einatmung derjenigen Luft, welche den 3-12 $\%$ Sauerstoff enthält, das $p H$ des Blutes zwar nach alkalischer Seite verschoben, während aber bei lang andauerndem $\mathrm{O}_{\mathrm{g}}$-Mangel die Azidität des Blutes schliesslich zunimmt.

Über die Frage, ob und inwieweit die Blutzirkulation im Gewebe, welche in wichtiger Beziehung zur Milchsäureresynthese steht, durch die Kohlensäurevergiftung, den Sauerstoffmangel und die geänderte Blutreaktion beeinflusst werde, treffen wir eine Fülle der literalischen Veröffentlichungen von Autoren an, wie Schwarz u. Lemberger, ${ }^{37}$ Fleisch, ${ }^{38)}$ Atzler u. Lehmann, ${ }^{39)}$ Atzler, ${ }^{40)}$ Hess, ${ }^{41}$ Schmitt, ${ }^{42}$ Iwai, ${ }^{43)}$ Nakagawa, ${ }^{4+}$ Hilton u. Eichholtz, ${ }^{45}$ Gremels u. Starling, ${ }^{46)}$ Eppinger, Kisch u. Schwarz. ${ }^{17}$ Dar-

27) Barcroft, Camis, Mathison, Roberts u. Ryffel, Philos. Transact. Roy. Soc. London, B, 1914, 206, 49 .

28) Lutz u. Schneider, Americ. Journ. Physiol., 1919, 50, 280.

29) H ald a ne, Brit. Mied. Jonrn., 1919, 3055, 65.

30) Haggard a. Henders on, Journ. Biol. Chem., 1920, 43, 3,

31) Macle od, Americ. Journ. Physiol., 1921, 55, 184.

32) Loewy, Pflüger's Arch., 1925, 207,632.

33) Kenster, Peemöller u. Schadow, Pflüger's Arch., 1927, 217, 473.

34) Yosomiy a, Tohoku Journ. Exp. Med., 1927, 8, 535.

35) Okad a, Gunidan Zasshi, 1930, 205, 999.

36) Gesell u. Hertzman, Proc. Soe. exper. Biol. a. Med., 1926, 24, 260.

37) Schwarz u. Lemberger, Pflüger's Arch., 1911, 141, 149.

38) Fleisch, Pflüger's Arch., 1918, 171, 86.

39) Atzler u. L ehmann, Pflüger's Arch., 1921, 190, 118.

40) Atzler, Deutsch. med. Wochenschr., 1923, 1011.

41) Hess, Ergebn. inn. Med. u. Kinderh., 1923, 23, 1.

42) Schmitt, Zeitschr. f. Biol., 1923, 79, 45.

43) Iwai, Pflüger's Arch., 1924, 202, 356.

44) N a k gawa, Pflüger's Arch., 1924, 203, 612.

45) Hilton u. Eichholtz, Journ. Physiol., 1925, 59, 413.

46) Gremels u. Starling, Journ. Physiol., 1926, 61, 297. 
unter hat $\mathrm{Fle}$ is $\mathrm{ch}^{38}$ ) nachgewiesen, dass die Kohlensäure, auf dieselbe Weise wie andere Säuren, in schwächeren Konzentrationen (wie z. B. $\frac{N}{1000}$ ) auf periphere Gefäse dilatierend, in stärkeren hingegen konstriktiv einwirkt. Hess ${ }^{4}$ ) gibt an, dass sowohl der $\mathrm{O}_{2}$-Mangel in der in Geweben zirkulierenden Flüssigkeit wie auch die Kohlensäureanhäufung, solange sie innerhalb der physiologischen Grenzen liegen, eine dilatatorische Wirkung habe, aber dam, wenn sie über dieselben hinaussteigen, eine konstriktorische Wirknng aufweise.

Was das Minutenvolum anbetrifft, ist die Zunahme desselben bei der $\mathrm{CO}_{2}$-Vergiftung bewiesen worden durch Jerusalem u. Starling, ${ }^{48}$ ) Mathison, ${ }^{49)}$ Itami, ${ }^{50)}$ Patterson, ${ }^{51)}$ Scott,${ }^{10)}$ Dale u. Evans, ${ }^{13)}$ Schneideru. Truesdell, ${ }^{51}$ ) Riml, ${ }^{59)}$ Gollwitzer-Meier, ${ }^{18)}$ Esveld, ${ }^{53}$ ) Grollmann, ${ }^{54}$ Schwiegk, $\left.{ }^{23}\right)$ Eppinger, Kisch u. Schwarz ${ }^{17}$ u. a.

Nach Beobachtungen von Harris on, Blalock, Pilcheru. Wilson, ${ }^{55}$ Grollmann, ${ }^{54)}$ Gollwitzer-Meier ${ }^{56)}$ und $\mathrm{Okada}^{35)}$ in hiesiger Klinik soll auch beim $\mathrm{O}_{2}$-Mangel eine Zunahme des Minutenvolnmens stattfinden.

Die Sanerstoffversorgung des Blutes auf Gewebe erleidet einen wesentlichen Einfluss durch die prozentuelle $\mathrm{O}_{2}$-Sättigung des Blutes, durch die Grösse der Geschwindigkeit der Oxydation und Reduktion des Blutes sowie durch die Blutzirkulation, und obige drei Faktoren stehen auch in inniger Beziehung zur Wasserstoffionenkonzentration. Bohr, Hasselbalch u. Krogh,57) Barcroft $u$. Camis, $\left.{ }^{58}\right)$ Barcroft $u$. Orbeli $i^{59}$ haben den Nachweis erbracht, dass wenn im Blut irgend welche Säuren, wie z. B. Kohlensäure oder Milchsäure, zunimmt, durch die Wirkung ihrer H-Ionen die $\mathrm{O}_{2}$-Bindungsfähigkeit des Hämoglobins vermindert und somit die Sauerstoffdissoziationskurve des Bluts erniedrigt ist. Mathison ${ }^{60)}$ machte die Beobachtung, dass die Gegenwart der organischen Säuren, wie Milchsäure, Essigsäure und Ameisensäure sowie der anorganischen Säuren, wie Salzsäure und Schwefelsäure u. a. die Geschwindigkeit der Reduktion arteriellen Blutes beschleunigt und dass auch die Anwesenheit der Kohlensäure eben dieselbe Wirkung wie oben erwähnte Säuren aufweist. Oinuma $a^{61)}$ hat nachgewiesen, dass die Gegenwart der Kohlensäure zu ver-

47) Hess, Die Regulierung des Blutkreislaufes, Leipzig 1930, 55.

48) Jerusale mu. Starling, Journ. Physiol., 1910, 40, 279.

49) Mathis on, Journ. Physiol., 1910, 41, 5.

50) It a mi, Journ. Physiol., 1912, 45, 338.

51) Patterson, Proc. Roy. Soc. London, 1914, 88, 371.

52) Riml, Schmiedeberg's Arch., 1929, 139, 240.

53) Esveld, Schmiedeberg's Arch., 1930, 147, 317.

54) Grollmann, Americ. Journ. Physiol,, 1930, 94, 287.

55) Harrison, Blalock, Pilcheru. Wilson, Americ. Journ. Physiol, 1987,

83, 284 .

56) Gollwitzer-Meier, Verh. Dtsch. Ges. inn. Med., 1929, 41, 361.

57) Bohr, Hasselbalch u. Krogh, Skand. Arch. f. Physiol., 1904, 16, 402.

58) B arcroft u. Camis, Journ. Physiol., 1909, 39, 118.

59) Barc roft u. Orbeli, Journ. Physiol., 1910, 41, 355.

60) Mathis o n, Journ. Physiol., 1911, 43, 347.

61) O in u ma, Journ. Physiol., 1911, 43, 364. 
mehrter Reduktion und verminderter Oxydation des Blutes führt. Hingegen gelang es $\left.\mathrm{Kato}{ }^{69}\right)$ experimentell nachzuweisen, dass durch Zusatz des Alkalis zum Blut die Geschwindigkeit der Oxydation grösser und dieselbe der Reduktion des Blutes kleiner wird; Momos $\mathrm{e}^{63)}$ beobachtete, dass der Zusatz von milchsaurem Natrium, kohlensaurem Natrium und dem Harnstoff zum Blut die Sauerstoff bindungsfähigkeit des Blutes mehr oder minder verstärkt. In hiesiger Klinik hat Ya maki ta ${ }^{64}$ an Kaninchen festgestellt, dass die Einspritzung des Natriumkarbonates die $\mathrm{O}_{2}$-Sättigung des Blutes sowie die $\mathrm{O}_{2}$-Dissoziationskurve zur Erhöhung bringt.

In Anbetiacht der Tatsache, dass Einflüsse, welche die Einatmung der kohlensäurereichen bzw. sauerstoffarmen Luft sowohl auf direktem wie auch indirektem Wege auf die Blutreaktion, Blutzirkulation und $\mathrm{O}_{2}-$ Versorgung im Organismus ausübt, eine so grosse Rolle, wie oben geschildert, spielen, glaube ich annehmen zu dürfen, dass die Einatmung derartiger Gase aus mannigfachen Gründen, wie sie eingangs angeführt wurden, den Umsatz der Milchsäure im Organismus, nämlich die Oxydation und Resynthese derselben in ausgedehntem Ausmasse beeinflussen muss. Demzufolge dürte auch angenommen werden, dass die Inhalation solcher Gase erhebliche Einflüsse auf die Ermüdung durch die Muskelarbeit sowie auf die Erholung davon ausuiben muss.

In vorliegender Abhandlung beschäftige ich mich mit Veränderungen des Milchsäurestoffwechsels, welche durch die Einatmung von dem Sauerstoff sowie der kohlensäurereichen bzw. sauerstoffarmen Luft bedingt sind.

\section{Versuchsmethode.}

Als Versuchsmaterial standen mir gesunde Kaninchen zur Verfügung. Zunächst habe ich, indem ich Kaninchen den Sauerstoff, die kohlensäurereiche bzw. sauerstoffarme Luft einatmen liess, ihnen bestimmte Mengen Milchsäure in die Vene injizierte und darauf geprüft, ob und mit welcher: Geschwindigkeit diese überschüssige Milchsäure im Organismus oxydativ synthetisiert werden mag.

$\mathrm{Zu}$ diesem $Z$ weck habe ich zuerst ein gesundes Kaninchen morgens früh in nüchternem Zustand im unmittelbaren Anschluss an die Gewichtsbestimmung an den elektrisch erwärmten Tierhalter gefesselt und ohne Vornahme der Narkose eine Kanüle zur Blutentnahme in die einseitige A. carotis eingeführt, und

\footnotetext{
62) T. K a to, Bioch. Journ., 1915, 9, 393.

63) Momose, Bioch. Journ, 1915, 9, 485.

64) Yamakita, Tohoku Jonrn. Exp. Med., 1921, 2, 290.
} 
behufs Gasinhalation eine Y-förmige, mit Klappen versehene Glaskanüle in die Trachea gelegt. Ungefähr 2 Stunden nach obigem Verfahren liess jch jedes Versuchstier das betreffende Gas in einem Spirometer aus dem einen Ende der Kanüle einatmen, während ich gleichzeitig die exspierte Luft in einem Sack von Dougl as, welcher mit einem anderen Ende derselben verbunden war, auffing; die so angesammelte Luft wurde dann der Analyse unterzogen.

Nach etwa $\frac{1}{3}$ stündiger Inhalation, wo der Einfluss der Gasinhalation manifest zutage trat, habe ich von einer 15\%igen Lösung des racemischen milch. sauren Natriums (Kahlbaum), die mit $\mathrm{NaOH}$ genügend neutralisiert war, 2,5 ccm pro $\mathrm{kg}$ Körpergewicht in einem Zeitraum von ca. 1,5 Minuten in die Ohrvene injiziert.

Damn habe ich in Intervallen von 5, 15, 30 und 60 Minuten darauf die Blutentuahme vorgenommen, die Milchsäure in so entnommenem Blut quantitativ bestimmt und sie auf die Verhältuisse der Oxydation und Resynthese untersucht. Gleichzeitig hiermit habe ich die Blut- $\mathrm{CO}_{\mathrm{g}}$ gemessen, um dadurch das Reservealkali des Blutes, also die Verschiebung der Veränderung der $\mathrm{cH}$ des Blutes zu würdigen. Weiterhin habe ich den $\mathrm{O}_{2}$-Verbrauch bei Versuchstieren bestimmt und somit auch deren Änderungen verfolgt.

Die Menge des jeweils entnommenen Blutes betrug ca. $2 \mathrm{ccm}$, es wurd 1 ccm davon zur Milchsäurebestiminung, die übrige Probe zur Bestimmung von Blut-CO benutzt.

Die quantitative Bestimmung der Milchsäure wurde nach der von Inawashiro und $\mathrm{Hayasaka^{65 } )}$ modifizierten Methode von $\mathrm{A}$ urep und Cannan aus. geführt, bei der Bestimmung von Blut- $\mathrm{CO}_{2}$ wurde der Apparat von van Slyke angewandt.

Der Sanerstoff verbranch wurde aus den in jeder Zeitspanne inspirierten Gasmengen sowie aus Vol. \% von in Ex- und Inspiration enthaltenen $\mathrm{O}_{2}$ und $\mathrm{CO}_{2}$ berechnet. Die Gasanalyse wurde mittels Haldan e's Apparates vorgenommen.

\section{Versuchsergebnisse.}

\section{Versuche bei der Einatmung von atmosphärischer Luft.}

Vorliegende Versuchsreiche wurde als Kontrollversuch für die Einatmung von Sauerstoff, sowie kohlensäurereicher bzw. sauerstoffarmer Luft ausgeführt, indem das Versuchstier die atmosphärische Luft eingeatmet hat. Das Ergebnis der diesbezüglichen 3 Versuche ist in Tab. 1 und Fig. 1 wiedergegeben.

Die Blut- $\mathrm{CO}_{2}$ hatte vor Laktatinjektion 45,7-49,3 Vol. \% betragen, während sie 5 Minuten danach bis auf 54,4-57,7 Vol. \% erhöht war, was also eine Zunahme um ca. $10 \mathrm{Vol}$. \% besagt. In Versuch 2 war die Blut- $\mathrm{CO}_{2}$ weiter nach 15 Ninuten müssig vermehrt, aber her-

65) In awashiro u. Hayasaka, Tohoku Journ. Exp. Med., 1928, 12, 1. 
Tabelle 1.

Versuch bei Laktatinjektion unter der Einatmung von atmosphärischer Luft.

\begin{tabular}{|c|c|c|c|c|c|c|c|c|}
\hline Nr. & 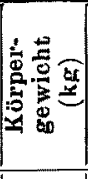 & $\begin{array}{c}\text { Zeit } \\
(\text { Min.) }\end{array}$ & 宽 & 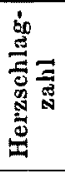 & 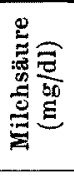 & 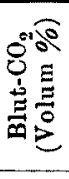 & 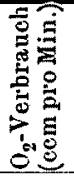 & Bemerkungen \\
\hline 1 & $\begin{array}{c}\mathbf{2 , 5} \\
(3)\end{array}$ & $\begin{array}{l}\text { Vor Inhalation } \\
\text { Vor Injektion } \\
5^{\prime} \text { nach } \\
15^{\prime} ", \\
30^{\prime} " \\
60^{\prime} "\end{array}$ & $\begin{array}{l}84 \\
88 \\
92 \\
84 \\
86 \\
84\end{array}$ & $\begin{array}{l}212 \\
222 \\
244 \\
240 \\
248 \\
222\end{array}$ & $\begin{array}{l}20,4 \\
21,3 \\
45,0 \\
35,6 \\
22,7 \\
18,6\end{array}$ & $\begin{array}{l}44,5 \\
45,7 \\
57,0 \\
55,0 \\
51,1 \\
46.9\end{array}$ & $\begin{array}{l}20,1 \\
29,8 \\
25,2 \\
\mathbf{2 5 , 0} \\
19,3\end{array}$ & $\begin{array}{l}6,2 \mathrm{ccm} 15 \% \text { Natr. } \\
\text { lact. i. v. (20 Min. } \\
\text { nach Beginn d. } \\
\text { Inhalation) }\end{array}$ \\
\hline 2 & $\begin{array}{c}2.4 \\
(7)\end{array}$ & $\begin{array}{l}\text { Vor Inhalation } \\
\text { Vor Injektion } \\
5^{\prime} \text { nach } \\
15^{\prime} " \\
30^{\prime} " \\
60^{\prime} "\end{array}$ & $\begin{array}{l}72 \\
72 \\
64 \\
60 \\
60\end{array}$ & $\begin{array}{l}260 \\
280 \\
272 \\
252 \\
264\end{array}$ & $\begin{array}{l}19,4 \\
42,4 \\
29,6 \\
20,6 \\
21,2\end{array}$ & $\begin{array}{l}49,3 \\
57,7 \\
58,6 \\
53,6 \\
50,5\end{array}$ & $\begin{array}{l}20,9 \\
30,3 \\
27,8 \\
27,0 \\
28,5\end{array}$ & $\begin{array}{l}6,0 \mathrm{ccm} 15 \% \text { Natr. } \\
\text { lact. i. v. }\end{array}$ \\
\hline $\mathbf{3}$ & $\begin{array}{r}2,0 \\
(\delta)\end{array}$ & $\begin{array}{l}\text { Vor Inhalation } \\
\text { Vor Injektion } \\
5^{\prime} \text { nach } \\
15^{\prime} " \\
30^{\prime} " \\
60^{\prime} "\end{array}$ & $\begin{array}{l}64 \\
66 \\
68 \\
72 \\
64 \\
68\end{array}$ & $\begin{array}{l}240 \\
240 \\
260 \\
272 \\
260 \\
252\end{array}$ & $\begin{array}{l}27,4 \\
26,0 \\
57,9 \\
46,3 \\
30,2 \\
25,6\end{array}$ & $\begin{array}{l}46,0 \\
46,9 \\
54,4 \\
48,7 \\
49,7 \\
46,9\end{array}$ & $\begin{array}{l}18,9 \\
26,3 \\
25,4 \\
20,1 \\
19,1\end{array}$ & $\begin{array}{l}5,0 \mathrm{ccm} 15 \% \text { Natr. } \\
\text { lact. i. v. }\end{array}$ \\
\hline \multicolumn{2}{|c|}{ Mittelwert } & $\begin{array}{l}\text { Vor Inhalation } \\
\text { Vor Injektion } \\
5^{\prime} \text { nach } \\
15^{\prime} " \\
30^{\prime} " \\
60^{\prime} "\end{array}$ & $\begin{array}{l}75 \\
77 \\
73 \\
70 \\
71\end{array}$ & $\begin{array}{l}241 \\
261 \\
261 \\
253 \\
246\end{array}$ & $\begin{array}{l}22,2 \\
48,4 \\
37,2 \\
24,5 \\
21,8\end{array}$ & $\begin{array}{l}47,3 \\
56,4 \\
54,1 \\
51,5 \\
48,1\end{array}$ & $\begin{array}{l}20,0 \\
28,8 \\
26,1 \\
24,0 \\
20,6\end{array}$ & \\
\hline
\end{tabular}

Fig. 1. Versuch bei Laktatinjektion unter der Inhalation von atmosphärischer Luft. (Versuch 1)

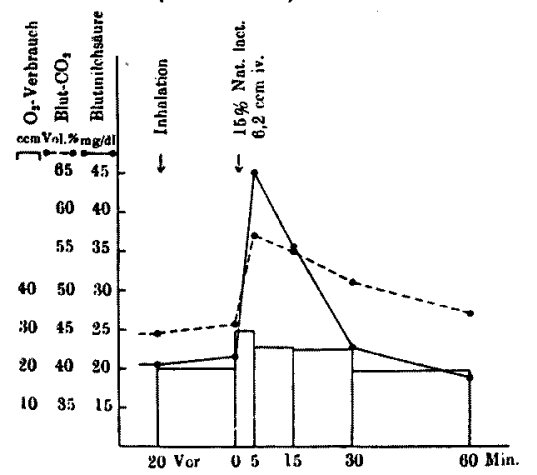

nach in allen Fällen allmählich vermindert, um nach 60 Minuten bis auf 46,9-50,5 Vol. \% abzusinken; also konnte sie hier sich zwar nicht vollkommen erholen, kam aber annähernd auf den früheren Wert vor Injektion zurück.

Die Blutmilchsäure betrug unmittelbar vor Injektion 19,4$26,0 \mathrm{mg} / \mathrm{dl}, 5$ Minuten danach war sie auf $42,4-57,9 \mathrm{mg} / \mathrm{dl} \mathrm{er}-$ höht, was eine Zunahme um 
23,0-31,9 mg/dl bedeutet. Von diesem Zeitpunkt an nahn sie jedoch allmählich ab, kam nach 30 Minuten nahe an den Ausgangswert zurück und erholte sich nach 60 Minuten in vollkommener Weise.

Der $\mathrm{O}_{2}$-Verbrauch betrug vor Laktatinjektion $18,9-20,9 \mathrm{ccm}$ pro Min., stieg aber in 5 Minuten nach Injektion auf 26,3-30,3 ccm; er zeigte also eine Zunahme um 7,4-9,7 ccm pro Min. Nunmehr kehrte er immer mehr abzunehmen um; bei Fall 1 erholte er sich in $60 \mathrm{Mi}-$ nuten vollkommen zum Ursprungswert, in übrigen 2 Fällen wies er noch im Ablauf von 60 Minuten eine mässige Zunahme auf.

Die Atemzahl blieb bei einem Fall konstant, bei den anderen Fällen hatte sie die Tendenz zur Zunahme, die aber nicht so bedeutend war. Die Pulszahl steigerte sich durch die Injektion deutlich.

Es ist seit Me yerh of $\left.{ }^{66}\right)$ allgemein gültig geworden, dass bei Kontraktion des Muskels als Quelle der Arbeitsenergie die Milchsäure aus in demselben enthaltenen Glykogen entsteht und dass die derartig entstandene Milchsäure bei der Erholung des betreffenden Muskels zu einem Teil daselbst verweilt, zu einem andern Teil auf dem Blutwege in andere ruhende Muskeln, ganz besonders aber in die Leber gelangt, wo ein Fünftel der gesamten Milchsäuremengen oxydiert wird, und ferner dass durch die Energie, die sich durch hierbei vor sich gehende Oxydationsprozesse entwickelt, die restliche Milchsäure zu Zucker resynthetisiert wird.

Die durch Muskelarbeit entstandene Milchsäure stellt die d-Milchsäure dar; weil es sich aber bei meinem Injektionsversuch um racemisches milchsaures Natrium handelte, möchte zwischen den beiden Milchsäurearten zwar irgendeiner biologischer Unterschied in bezug auf die Geschwindigkeit der Diffusion ins Gewebe und dergleichen bestehen, im Zusammenhang damit ist es nicht zu leugnen, dass das von mir injizierte Laktat in bezug auf die Fähigkeit zur Resynthese gegenüber der durch Körperarbeit entstehende Milchsäure nicht gerade isodynam sich verhielt, dennoch ist soviel als sichergestellt anzusprechen, dass ein Anteil von injiziertem Laktat durch den Sauerstoff oxydiert und ein anderer Anteil resynthetisiert worden ist. Und weil die durch die Oxydation entstandene Kohlensäure sich mit dem von dem Laktat freigewordenen Na-Ion verbindet, dürfte es zur' Vermehrung des Reservealkalis im Blut kommen.

Es ist nun noch weiter festgestellt worden, dass die Milchsäureresynthese bei der Einatmung der atmosphärischen Luft in 30-60 Minuten aufhört.

66) Megerhof, Pfl 1 üger's Arch., 1920, 185, 25. 


\section{Versuche bei der Sauerstoffinhalation.}

Demnächst wurde, nachdem man Versuchstieren unterSauerstoffinhalation das Laktat injiziert hatte, denselben Versuch wie der vorige Versuch ausgeführt. Behufs Sauerstoffeinatmung wurde der Respirationsapparat von Sanborn-Benedict angewandt. Die Ergebnisse in 6 Versuchen werden aus Tab. 2 und Fig. 2 veranschaulicht.

Die Blutkohlensäure hatte vor $\mathrm{O}_{2}$-Einatmung $42,2-49,5 \mathrm{Vol} . \%$, im Mittel 45,7 Vol. \% betragen. 20 Minuten nach Eröffnung der $\mathrm{O}_{2}$ -

Tabelle 2.

Versuch bei Laktatinjektion unter der Einatmung von reinem Sauerstoff.

\begin{tabular}{|c|c|c|c|c|c|c|c|c|}
\hline $\mathrm{Nr}$. & 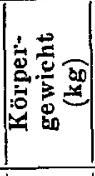 & $\begin{array}{c}\text { Zeit } \\
\text { (Min.) }\end{array}$ & 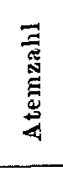 & 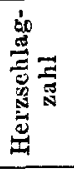 & 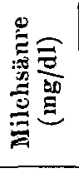 & 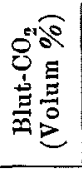 & 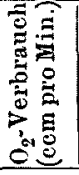 & Bemerkungen \\
\hline 1 & $\begin{array}{l}1,85 \\
(\delta)\end{array}$ & $\begin{array}{l}\text { Vor Inhalation } \\
\text { Vor Injektion } \\
5^{\prime} \text { nach } \\
15^{\prime} " \\
30^{\prime} " \\
60^{\prime} "\end{array}$ & $\begin{array}{l}76 \\
74 \\
80 \\
76 \\
72 \\
76\end{array}$ & $\begin{array}{l}244 \\
240 \\
266 \\
258 \\
252 \\
240\end{array}$ & $\begin{array}{l}32,1 \\
33,0 \\
51,7 \\
\mathbf{4 6 , 0} \\
31,9 \\
\mathbf{2 8 , 5}\end{array}$ & $\begin{array}{l}49,5 \\
49,5 \\
59,9 \\
57,1 \\
57,9 \\
48,9\end{array}$ & $\begin{array}{l}20,6 \\
36,0 \\
30,6 \\
26,6 \\
24,8\end{array}$ & $\begin{array}{l}4,7 \mathrm{ccm} 15 \% \text { Natr. } \\
\text { lact. i. v. }\end{array}$ \\
\hline 2 & $\begin{array}{l}1,87 \\
(ㅇ ㅜ)\end{array}$ & $\begin{array}{l}\text { Vor Inhalation } \\
\text { Vor Injektion } \\
5{ }^{\prime} \text { nach } \\
15^{\prime} " \\
30^{\prime} " \\
60^{\prime} "\end{array}$ & $\begin{array}{l}56 \\
60 \\
64 \\
64 \\
60 \\
60\end{array}$ & $\begin{array}{l}200 \\
204 \\
264 \\
264 \\
240 \\
212\end{array}$ & $\begin{array}{l}27,6 \\
26,0 \\
66,2 \\
60,5 \\
32,4 \\
33,1\end{array}$ & $\begin{array}{l}45,2 \\
46,1 \\
54,5 \\
48,9 \\
48,9 \\
41,7\end{array}$ & $\begin{array}{l}18,8 \\
28,3 \\
21,3 \\
18,8 \\
20,0\end{array}$ & $\begin{array}{l}4,7 \text { ccm } 15 \% \text { Nats. } \\
\text { lact. i. v. }\end{array}$ \\
\hline $\mathbf{3}$ & $\begin{array}{l}2,08 \\
(q)\end{array}$ & $\begin{array}{l}\text { Vor Inhalation } \\
\text { Vor Injektion } \\
5^{\prime} \text { nach } \\
155^{\prime} " \\
30^{\prime} " \\
60^{\prime} "\end{array}$ & $\begin{array}{r}80 \\
80 \\
82 \\
80 \\
76 \\
76\end{array}$ & $\begin{array}{l}240 \\
224 \\
264 \\
260 \\
252 \\
240\end{array}$ & $\begin{array}{l}23,4 \\
24,9 \\
48,5 \\
35,6 \\
26,6 \\
22,5\end{array}$ & $\begin{array}{l}42,8 \\
44,7 \\
51,3 \\
48,5 \\
44,4 \\
44,4\end{array}$ & $\begin{array}{l}24,9 \\
42,1 \\
37,3 \\
33,8 \\
29,0\end{array}$ & $\begin{array}{l}5,2 \text { cem } 15 \% \text { Natr. } \\
\text { lact. i. } v \text {. }\end{array}$ \\
\hline 4 & $\begin{array}{l}2,0 \\
(9)\end{array}$ & $\begin{array}{l}\text { Vor Inhalation } \\
\text { Vor In,jektion } \\
5^{\prime} \text { nach } \\
15^{\prime} " \\
30^{\prime} " \\
60^{\prime} "\end{array}$ & $\begin{array}{l}72 \\
72 \\
72 \\
76 \\
72 \\
72\end{array}$ & $\begin{array}{l}270 \\
262 \\
288 \\
276 \\
264 \\
260\end{array}$ & $\begin{array}{l}18,1 \\
16,1 \\
40,2 \\
37,9 \\
16,1 \\
18,0\end{array}$ & $\begin{array}{l}42,2 \\
42,2 \\
49,9 \\
40,3 \\
42,2 \\
42,2\end{array}$ & $\begin{array}{l}26,5 \\
37,2 \\
34,0 \\
27,5 \\
26,7\end{array}$ & $\begin{array}{l}5,0 \mathrm{ccm} 15 \% \text { Natr. } \\
\text { lact. } 1 . \mathrm{v} .\end{array}$ \\
\hline $\mathbf{5}$ & $\begin{array}{l}2,1 \\
(\%)\end{array}$ & $\begin{array}{l}\text { Vor Inhalation } \\
\text { Vor Injektion } \\
5^{\prime} \text { nach } \\
15^{\prime} " \\
30^{\prime} " \\
60^{\prime} "\end{array}$ & $\begin{array}{l}60 \\
68 \\
64 \\
64 \\
60 \\
60\end{array}$ & $\begin{array}{l}280 \\
288 \\
294 \\
294 \\
288 \\
288\end{array}$ & $\begin{array}{l}16,1 \\
18,0 \\
46,3 \\
31,9 \\
18,4 \\
17,6\end{array}$ & $\begin{array}{l}47,3 \\
45,4 \\
49,1 \\
49,1 \\
49,1 \\
47,3\end{array}$ & $\begin{array}{l}20,5 \\
24,8 \\
23,3 \\
22,0 \\
19,2\end{array}$ & $\begin{array}{l}5,25 \mathrm{ccm} 15 \% \mathrm{Natr} \text {. } \\
\text { lact. i. v. }\end{array}$ \\
\hline
\end{tabular}




\begin{tabular}{|c|c|c|c|c|c|c|c|c|}
\hline Nr. & 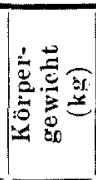 & $\begin{array}{c}\text { Zeit } \\
\text { (Min.) }\end{array}$ & 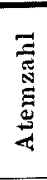 & 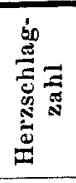 & 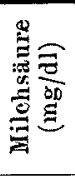 & 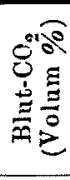 & 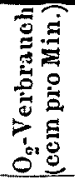 & Bemerkungen \\
\hline 6 & $\begin{array}{l}2,08 \\
(\hat{\delta})\end{array}$ & $\begin{array}{l}\text { Vor Inhalation } \\
\text { Vor Injektion } \\
5^{\prime} \text { nach } \\
15^{\prime} " \\
30^{\prime} " \\
60^{\prime} ",\end{array}$ & $\begin{array}{l}68 \\
64 \\
72 \\
64 \\
64 \\
72\end{array}$ & $\begin{array}{l}204 \\
200 \\
236 \\
236 \\
222 \\
214\end{array}$ & $\begin{array}{l}14,9 \\
16,9 \\
37,6 \\
30,2 \\
18,0 \\
13,5\end{array}$ & $\begin{array}{l}47,1 \\
47,1 \\
50,8 \\
52,7 \\
50,8 \\
45,2\end{array}$ & $\begin{array}{l}17,5 \\
20,4 \\
19,4 \\
18,1 \\
14,6\end{array}$ & $\begin{array}{l}5,2 \mathrm{ccm} 15 \% \text { Natr. } \\
\text { lact. i. v. }\end{array}$ \\
\hline Mitt & elwert & $\begin{array}{l}\text { Vor Inhalation } \\
\text { Vor Injektion } \\
5^{\prime} \text { nach } \\
15^{\prime} " \\
30^{\prime} " \\
60^{\prime} "\end{array}$ & $\begin{array}{l}69 \\
70 \\
72 \\
71 \\
67 \\
69\end{array}$ & $\begin{array}{l}240 \\
236 \\
269 \\
265 \\
253 \\
242\end{array}$ & $\begin{array}{l}22,0 \\
22,5 \\
48,4 \\
40,4 \\
23,9 \\
22,2\end{array}$ & $\begin{array}{l}45,7 \\
45,8 \\
52,6 \\
49,4 \\
48,9 \\
45,0\end{array}$ & $\begin{array}{l}21,5 \\
31,5 \\
27,7 \\
24,5 \\
22,4\end{array}$ & \\
\hline
\end{tabular}

Fig 2. Versuch bei der Laktatinjektion nuter der Inhalation von Sauerstoff. (Ver'such 3)

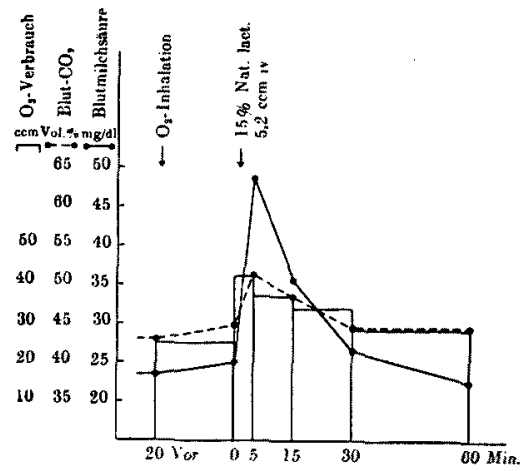

Inhalation, nämlich unmittelbar vor Laktatinjektion betrug die Blut- $\mathrm{CO}_{2} 42,2-49,5$ Vol. \%, durclischnittlich $45,8 \mathrm{Vol} . \%$, es wurde also fast keine Änderung der Blut- $\mathrm{CO}_{2}$ durch die $\mathrm{O}_{2}$-Einatmung hervorgerufen. In 5 Minuten nach Laktatinjektion zeigte sie $49,1-59,9 \%$, im Durchschnitt 52,6\%, eine Zunahme um 3,7-10,4 Vol. \%. Diese Zunahme ist aber etwas kleiner als die des in atmosphärischer Luft befindlichen Kontrolltieres. Nach

diesem Abschnitt nahm die Blut- $\mathrm{CO}_{2}$ nur bei Versuch 6 nach 15 Minuten von neuem ein wenig zu, um sich nach 60 Minuten zum Anfangswert zu erholen, während sie bei anderen 6 Fällen im Ablauf von 5 Minuten nach initialer. Zunahme allmählich abnahm und nach 30-60 Minuten ganz auf den Wert, der unmittelbar vor Injektion gefunden wurde, zurïckkam.

Die Blutmilchsäure hatte vor Injektion $14,9-32,1 \mathrm{mg} / \mathrm{dl}$, im Mittel $22,0 \mathrm{mg} / \mathrm{dl}$ betragen, in 20 Minuten nach $\mathrm{O}_{\mathrm{g}}$-Inhalation betrug sie $16,1-33,0 \mathrm{mg} / \mathrm{dl}$, im Durchschnitt 22,5 mg/dl. Bei jedem einzelnen Fall betrachtet, zeigte sie Schwankung von nur um $2 \mathrm{mg}$ herum; diese Schwankung wäre nicht als durch den Einfluss der $\mathrm{O}_{2}$-Einatmung bedingt, sondern eher als physiologische anzusehen. In 5 Minuten nach 
Laktatinjektion wurde die Milchsäure zu einem Wert von 37,6-66,2 $\mathrm{mg} / \mathrm{dl}$, also zu einem Mittelwert von $48,4 \mathrm{mg} / \mathrm{dl}$ gefunden, hier zeigte sich nämlich eine Zunahme um 18,7-40,2, durchschnittlich $25,9 \mathrm{mg} / \mathrm{dl}$; sie war nahezu in glcichem Masse vermehrt, wie die beim Kontrolltier ermittelte Zunahme um durchschnittlich $24,5 \mathrm{mg} / \mathrm{dl}$. Alsdann nahm die Milchsäure graduell ab, bis sie nach 30 Minuten schliesslich bei der Hälfte der Fälle bis auf den vor Injektion ermittelten Wert wiederhergestellt war. In anderen Fällen erholte sie sich nach 30 Minuten wenn auch nicht vollkommen, so doch annähernd, nach 60 Minuten völlig zum. Ursprungswert. Bei Versuch 2 allein, der die höchste Steigeiung der Milchsäure aufgewiesen hatte, behielt dieselbe selbst nach 30 Minuten noch eine Zunahme um $6 \mathrm{mg}$ bei, nahm nach 60 Minuten von neuem gewissermassen $\mathrm{zu}$, ohne sich erholt zu haben.

Der $\mathrm{O}_{3}$-Verbrauch betrug während der Sauerstoffinhalation 17,526,5, im Mittel 21,5 ccm pro Min., binnen eines Zeitraumes von $5 \mathrm{Mi}$ nuten nach Laktatinjektion betrug er 22,4-42,1 ccm, im Mittel 31,5 ccm, das war eine Zunahme um 3-20 ccm, durchschnittlich um $10 \mathrm{ccm}$. Dieser Betrag gilt als eine geringe Zunahme gegenüber der Zunahme un $8,8 \mathrm{ccm}$ beim Kontrolltier. Von diesem Zeitpunkt an nahm der 0.-Verbrauch allmählich ab, und zwar bei Versuch 2 nach 30 Minuten, bei Versuch 5 und 6 nach 60 Minuten kam er bereits auf den Anfangswert zurück. In anderen 3 Versuchen nahm er nach 60 Minuten zu dem wenn auch nicht vollkommenen, doch annähernden Ursprungswert ab.

Die Atemzahl liess durch die $\mathrm{O}_{\mathrm{g}}$-Einatmung allein keine merkliche Änderung erkennen, obzwar sie hierbei mehr oder weniger Schwankungen unterworfen war. Durch die Laktatinjektion war sie entweder unverändert oder einigermassen vermehrt, die vermehrte Atemzahl reduzierte sich aber danach allmählich und kam schliesslich auf die frühere Zahl zurück. Die Pulszahl hat durch die $\mathrm{O}_{2}$-Einatmung eine geringe Schwankung dar, war durch die Laktatinjektion deutlich erhöht und darauf allmählich herabgesetzt.

Oben geschilderte, bei der $\mathrm{O}_{\mathrm{a}}$-Einatmung durch die Laktatinjektion gewonnene Ergebnisse in bezug auf den Verlauf von Blut- $\mathrm{CO}_{2}$, Milchsäure und $\mathrm{O}_{2}-$ Verbrauch stiminen mit dem Ergebnis von $\mathrm{H}$ ay asaka $a^{(i)}$ gut überein.

Bielschowsky und Thaddea $\mathbf{a}^{(8)}$ machten die Beobachtung, dass die Nüchtern-Milchsäurespiegel bei der Inhalation reinen Sanerstoffes bei gesun-

67) Hay as ak a, Tohoku Journ. Exp. Med., 1929, 14, 359.

68) Bielschowsky u. Thadden, Zeitschr. kl. Med., 1932, 120, 330. 
den Menschen unbeeinflusst blieb, und dass im Belastungsversuch mit Milchsäure in der Form der Injektion von $10-40 \mathrm{ccm} \mathrm{20 \% iger} \mathrm{Natriumlaktatlösung}$ der Milchsäurespiegel bei der Sauerstoffeinatmung bedeutend niedriger als bei der Einatmung von atmosphärischer Luft war. Hill und Mackenzie ${ }^{(9)}$ geben an, dass man bei Muskelarbeit, vor der die $\mathrm{O}_{\mathrm{a}}$-Inhalation vorgenommen worden war, die Arbeit mit Leichtigkeit zu leisten vermöge, Hill, Long und Lupton $n^{70}$ haben darauf hingewiesen, dass bei körperlicher Arbeit, die bei der Einatmung des 50\% Sauerstoffes geleistet wird, die während der Arbeit erfolgende $\mathrm{O}_{2}$-Aufnahme ausserordentlich gesteigert sei, und dass auch bezüglich der Erholungsperiode die Erholung im ersten Stadium beschleunigt, im zweiten Stadium aber nicht weiter beschleunigt werde.

Während dies-nach Angaben obiger Autoren-bei der Einatmung von 50 $\% \mathrm{O}_{2}$ der Fall ist, soll bei der Einatmung von 100\% $\mathrm{O}_{2}$ der Einfluss nicht mehr so deutlich in Erscheinung treten. Hewlett, Barnett und Lewis ${ }^{71}$ konnten nachweisen, dass bei Arbeit unter der Einatmung der sauerstoffreichen Luft die Milchsäure im Blut und Harn im Vergleich mit derselben unter der Einatmung der gewöhnlichen Luft vermindert war. Fischer-Wasels und Büngele ${ }^{* 2}$ ) konstatierten, dass die $\mathrm{O}_{2}$-Einatmung keinen Einfluss auf den Sauerstoff verbrauch in der Ruhe ausübte.

In meinem Versuche konnte ich durch die Einatmung reinen Sauerstoffes allein keine nennenswerten Änderungen in bezug auf die Blut$\mathrm{CO}_{2}$, Blutmilchsäure und den $\mathrm{O}_{\mathrm{g}}$-Verbrauch konstatieren. Auch die nach Laktatinjektion aufgetretene Vermehrung und Erholung von Blut- $\mathrm{CO}_{2}$ und Blutmilchsäure zeigten fast keinen Unterschied im Vergleich mit denselben bei Kontrolltieren. Der $\mathrm{O}_{2}$-Verbrauch war in der Mehrzahl der Fälle in direktem Anschluss an die Laktatinjektion ziemlich mehr vermehrt als derselbe bei Kontrolltieren, hinsichtlich der Zeitdauer aber, die zur Erholung zu seinem Ursprungswert in Anspruch genommen wurde, bestand kein Unterschied zwischen Versuchs- und Kontrolltieren.

Aus dem Umstand, dass die $\mathrm{O}_{2}$-Einatmung keine Änderung von Blut- $\mathrm{CO}_{2}$ bewirkt, dürfte wohl geschlossen werden, dass sie auch keinen Einfluss auf die Blutreaktion ausübt, und dass, weil die $\mathrm{O}_{2}$ Sättigung des Blutes beim Blut von normalem cH-Gehalt jedenfalls, selbst wenn der $\mathrm{O}_{2}$-Gehalt der Inspirationsluft nur $20 \%$ beträgt oder der annähernd reine Sauerstoff inspiriert wird, nur einen geringen Unterschied aufweist, der hierzu erforderliche Sauerstoff aus atmosphärischer Luft zur Genüge aufgenommen werden kann.

69) Hill a. Mackenzie, Journ. Physiol., 1909-10, 39, 33.

70) Hill, Long u. Lu pto n, Proc. Roy. Soc. London, 1925, 97, 84

71) Hewlet t, Barnett u. Lew is, Jonrn. Clinic. Invest, 1926, 3, 317.

72) Fischer-Wasels u. Bitngeler, Frankfurter Zeitschr. f. Pathol., 1930, 39, 288. 


\section{Versuche bei der Inhalation von kohlensäurereicher Luft.}

1. Inhalation von kohlensäurereicher Luft ohne die Laktatinjektion.

In dieser Versuchsreihe musste das Kaninchen eine Luft, die 7-10 $\% \mathrm{CO}_{2}$ und ca. $21 \% \mathrm{O}_{2}$ enthielt, inspirieren. Das Ergebnis in $5 \mathrm{Ver}-$ suchen ist in Tab. 3 u. Fig. 3 dargestellt.

Während die Blutkohlensäure vor der Inhalation 43,0-50,3, im Mittel 47,3 Vol. \% betrug, wurde in 20 Minuten danach zu 53,5- 72,9 $\%$, also zu einem Durchschnittswert von 59,1 Vol. \% gefunden; hier trat also eine bedeutende Zunahme auf. Diese Zunahme setzte sich noch weiter nach 25,50 und 80 Minuten fort, und zwar bis zum Ablauf von 20 Minuten erfolgte sie mit raschem Schritt, von diescin Zeitpunkt an war die Zunahme eher sehr verzögert. In einem (Ver-

Tabelle 3.

Versuch unter der Einatmung von kohlensäurereicher Luft.

\begin{tabular}{|c|c|c|c|c|c|c|c|c|}
\hline Nr. & 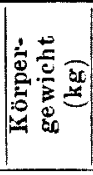 & $\begin{array}{c}\text { Zeit } \\
\text { (Min.) }\end{array}$ & 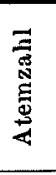 & 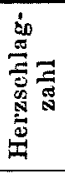 & 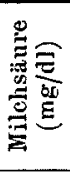 & 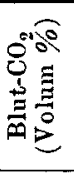 & 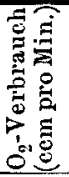 & Bemerkungen \\
\hline 1 & $\begin{array}{l}1,8 \\
(ㅇ)\end{array}$ & $\begin{array}{l}\text { Vor Inhalation } \\
20^{\prime} \text { nach } \\
25^{\prime} " \\
35^{\prime} " \\
50^{\prime} " \\
80^{\prime} "\end{array}$ & $\begin{array}{l}72 \\
80 \\
84 \\
86 \\
92 \\
92\end{array}$ & $\begin{array}{l}270 \\
188 \\
188 \\
194 \\
194 \\
200\end{array}$ & $\begin{array}{l}29,6 \\
26,4 \\
25,0 \\
21,9 \\
21,7 \\
16,7\end{array}$ & $\begin{array}{l}50,3 \\
72,9 \\
70,8 \\
67,1 \\
67,1 \\
65,2\end{array}$ & $\begin{array}{l}16,6 \\
19,0 \\
19,5 \\
21,4 \\
22,1\end{array}$ & $\begin{array}{c}\mathrm{CO}_{2} 10,4 \% \\
\mathrm{O}_{2}^{-2} 20,6 \%\end{array}$ \\
\hline 2 & $\begin{array}{l}1,7 \\
(\hat{\delta})\end{array}$ & $\begin{array}{l}\text { Vor Inhalation } \\
20^{\prime} \text { nach } \\
25^{\prime} " \\
35^{\prime} " \\
50^{\prime} " \\
80^{\prime} "\end{array}$ & $\begin{array}{r}76 \\
102 \\
96 \\
96 \\
96 \\
96\end{array}$ & $\begin{array}{l}292 \\
222 \\
228 \\
228 \\
246 \\
264\end{array}$ & $\begin{array}{l}30,7 \\
24,7 \\
25,1 \\
21,2 \\
18,0 \\
19,4\end{array}$ & $\begin{array}{l}50,0 \\
57,4 \\
61,1 \\
62,0 \\
62,9 \\
64,8\end{array}$ & $\begin{array}{l}20,1 \\
20,8 \\
21,1 \\
20,7 \\
19,7\end{array}$ & $\begin{array}{c}\mathrm{CO}_{2} \quad 8,1 \%, \\
\mathrm{O}_{2} 21,1 \%\end{array}$ \\
\hline 3 & $\begin{array}{l}2,31 \\
(\delta)\end{array}$ & $\begin{array}{l}\text { Vor Inhalation } \\
20^{\prime} \text { nach } \\
25^{\prime} " \\
35^{\prime} " \\
50^{\prime} " \\
80^{\prime} "\end{array}$ & $\begin{array}{l}70 \\
88 \\
92 \\
92 \\
92 \\
88\end{array}$ & $\begin{array}{l}246 \\
164 \\
172 \\
176 \\
180 \\
180\end{array}$ & $\begin{array}{l}20,3 \\
15,8 \\
15,8 \\
14,9 \\
12,6 \\
10,8\end{array}$ & $\begin{array}{l}43,0 \\
53,8 \\
53,8 \\
55,5 \\
56,4 \\
57,3\end{array}$ & $\begin{array}{l}16,1 \\
15,6 \\
16,2 \\
16,7 \\
16,2\end{array}$ & $\begin{array}{c}\mathrm{CO}_{2} \quad 8,9 \%, \\
\mathrm{O}_{2} 21,0 \%\end{array}$ \\
\hline 4 & $\begin{array}{l}2,1 \\
(q)\end{array}$ & $\begin{array}{l}\text { Vor Inhalation } \\
20^{\prime} \text { nach } \\
25^{\prime} " \\
35^{\prime} " \\
50^{\prime} " \\
80^{\prime} "\end{array}$ & $\begin{array}{l}56 \\
88 \\
88 \\
92 \\
88 \\
88\end{array}$ & $\begin{array}{l}240 \\
200 \\
170 \\
164 \\
204 \\
204\end{array}$ & $\begin{array}{l}26,4 \\
17,4 \\
16,7 \\
17,4 \\
14,9 \\
10,4\end{array}$ & $\begin{array}{l}47,2 \\
58,1 \\
56,3 \\
58,1 \\
59,9 \\
59,9\end{array}$ & $\begin{array}{l}18,5 \\
18,1 \\
17,5 \\
19,1 \\
19,3\end{array}$ & $\begin{array}{c}\mathrm{CO}_{2} \quad 7,4 \% \\
\mathrm{O}_{2} 21,1 \%\end{array}$ \\
\hline
\end{tabular}




\begin{tabular}{|c|c|c|c|c|c|c|c|c|}
\hline $\mathrm{Nr}$ & 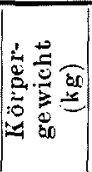 & $\begin{array}{c}\text { Zeit } \\
\text { (Min.) }\end{array}$ & 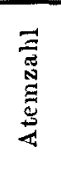 & 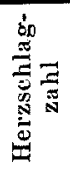 & 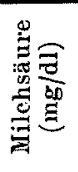 & 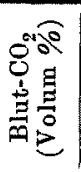 & 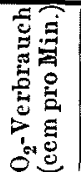 & Bemerkungen \\
\hline 5 & $\begin{array}{l}1,8 \\
(9)\end{array}$ & $\begin{array}{l}\text { Vor Inhalation } \\
20^{\prime} \text { nach } \\
25^{\prime}, " \\
35^{\prime} ", \\
50^{\prime} ", \\
80^{\prime} ",\end{array}$ & $\begin{array}{r}66 \\
112 \\
120 \\
114 \\
114 \\
114\end{array}$ & $\begin{array}{l}246 \\
200 \\
208 \\
200 \\
258 \\
258\end{array}$ & $\begin{array}{l}24,4 \\
19,9 \\
18,6 \\
17,5 \\
14,9 \\
12,2\end{array}$ & $\begin{array}{l}46,2 \\
53,5 \\
55,4 \\
55,4 \\
57,2 \\
59,1\end{array}$ & $\begin{array}{l}16,6 \\
16,2 \\
16,6 \\
15,9 \\
16,4\end{array}$ & $\begin{array}{c}\mathrm{CO}_{2} 8,4 \%, \\
\mathrm{O}_{2} 20,7 \%\end{array}$ \\
\hline \multicolumn{2}{|c|}{ Mittelwert } & $\begin{array}{l}\text { Vor Inhalation } \\
20^{\prime} \text { nach } \\
25^{\prime}, " \\
35^{\prime} ", \\
50^{\prime} ", \\
80^{\prime} ",\end{array}$ & $\begin{array}{l}68 \\
94 \\
96 \\
96 \\
96 \\
96\end{array}$ & $\begin{array}{l}2 \tilde{2} 9 \\
195 \\
193 \\
192 \\
216 \\
221\end{array}$ & $\begin{array}{l}26,3 \\
20,8 \\
20,2 \\
18,6 \\
16,4 \\
13,9\end{array}$ & $\begin{array}{l}47,3 \\
59,1 \\
59,5 \\
59,6 \\
60,7 \\
61,3\end{array}$ & $\begin{array}{l}17,6 \\
17,9 \\
18,2 \\
18,8 \\
18,7\end{array}$ & \\
\hline
\end{tabular}

Fig. 3. Versuch nnter der Inhalation von kohlensäurereicher Luft. (Versuch 5)

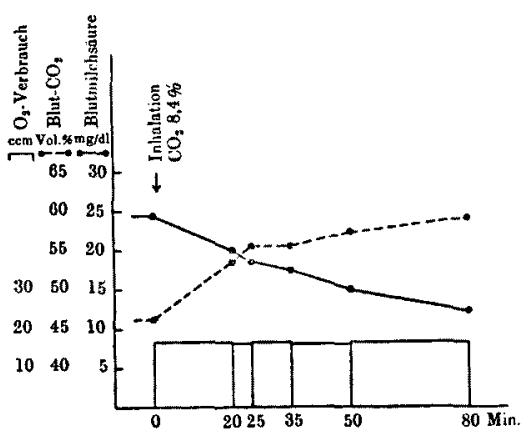

such 1) von 5 Fällen erreichte Blut- $\mathrm{CO}_{2}$ in 20 Minuten das Maximum und war darauf vielmehr geneigt, langsam abzunehmen, in Versuch 4 nahm sie im Ablauf von 25 Min. einmal ab und danach wieder zu.

Die Blutmilchsäure war vor der Inhalation bei $20,3-30,7$, im Mittel bei $26,3 \mathrm{mg} / \mathrm{dl}$ gelegen, mit Eröffnung der Einatmung von Kohlensäure nahm sie derart $a b$, dass sie nach 20 Minuten 15,8-26,4, im Mittel 20,8 mg/dl betrug; nach 25 Minuten war sie, während sie in Versuch 2 und 3 konstant blieb, in anderen 3 Versuchen noch gewissermassen vermindert, nach 35 Minuten nahm sie, mit Ausnahme von Versuch 4 mit weiterer geringer Zunahme, fortlaufend ab. Von da ab bis zum Ablauf von 80 Minuten nahm die Blutmilchsäure in allen Fällen kontinuierlich graduell ab, bis sie nach 80 Minuten einen Wert von 10,4-19,4, einen Durchschnittswer't von $13,9 \mathrm{mg} / \mathrm{dl}$ erreichte.

Der $\mathrm{O}_{2}$-Verbrauoh wurde in 20 Minuten nach Eröffnung der Inhalation zu 16,1-20,1, im Mittel zu 17,6 ccm pro Min. gefunden. Dieser Wert erwies sich als etwas kleiner gegenüber einem Durchschnittswert von $20,0 \mathrm{~cm}$ bei der Einatmung von normaler Luft sowie in Vergleich zu einem Mittelwert von 21,5 ccm bei der Einatmung von Sauerstoff. 
Nach diesem Zeitschnitt verluarrte der $\mathrm{O}_{2}$-Verbrauch in wesentlichen auf demselben Betrag, nun geringe Schwankung ron $1 \mathrm{ccm}$ herum aufweisend ; nur bei Versuch 1, wo $\mathrm{cr}$ anfünglich $16.6 \mathrm{ccm}$ betragen hatte, nahm er immer melhr um ein geringes zu und erreichte nach 80 Minuten einen Wert von $22,1 \mathrm{ccm}$.

Die Atemzahl wurde in allen Fiillen durch Inhalation von Kohlensäure gesteigert, diese Steigerung fiel jedoch je nach den Fällen verschieden aus, im grossen und ganzen aber zeigte eine Zunahme um ca. 20. Die Herzschlagzahl nahm durch Inhalation von Kohlensäure um 40-80 ab, in den meisten Fällen nahm sie fortlaufend bis zum Abschluss der Inhalation ab, während sie bei Versuch 5 nach 50-80 Minuten etwas vermehrt gefunden wurde.

Wie bereits in der Einleitung erwähnt, liegt es auf der Hand, dass bei der Inhalation von kohlensäurereicher Luft die alveolare $\mathrm{CO}_{:^{-}}$ Spannung erhöht ist, wodurch die $\mathrm{CO}_{2}$-Diffusion aus dem Blut behindert wird und infolgedessen die $\mathrm{CO}_{\mathrm{z}}$-Retention in Blut und Gewebe zustande kommt. Dass die Blutkohlensäure im Anfangsstadium der Inhalation von Kohlensäure mit einem Schlag und dann verzögert zunimmt, ist darauf zurückzuführen, dass die Kohlensäure zum Teil sich mit dem in Blut und Gewebsflüssigkeit vorhandenen Reservealkali verbindet und zum anderen Teil, weil sie in der Gewebsflüssigkeit leicht löslich ist, sich physikalisch darin rasch auföst, und dass wenn die Blutkohlensäure eine bestimmte Höhc erreicht hat, die weitere $\mathrm{Zu}$ nahme nur langsam erfolgen kann.

Über den Einfluss der Einatmung von kohlensäurereicher Luft auf den Blutmilchsäurespiegel liegen zahlreiche Arbeiten vor.

$\mathrm{Hochrein}$ und Meie $\mathrm{r}^{\mathrm{r} 3}$ haben an gesunden Menschen durch die 5 Minuten lange Einatmung eines Gasgemisches von $5 \% \mathrm{CO}_{2}$ und $95 \% \mathrm{O}_{2}$ ausnahmslos eine geringe Zunahme des Milchsäurespiegels festgestellt und diese auf die Vermehrung der Wasserstoffionen durch die Respiration von Kohlensäure zurückgeführt. Im Gegensatz hierzu sahen Anrep u. Cann a nit) im Versuch an „Heart-lung-preparation" bei der Einatmung der 7,2-9,4\% $\mathrm{CO}_{2}$ haltigen Luft ausgesprochene Azidosis und eine Senkung des Blutmilchsäurespiegels auftreten, auch $\mathrm{L}$ ong ${ }^{-55}$ konstatierte bei halbstündiger Einatmung von $8-11 \% \mathrm{CO}_{2}$ eine Abnahme der Milchsäure, diese Veründerung hat er der Vermehrung der $\mathbf{H}^{*}$-Konzentration zugeschrieben. In hiesiger Klinik haben Hay as aka u. I takura ${ }^{76}$ am Menschen durch die 5-8 Minuten anhaltende Eihatmung von 5,3-

73) Hochrein u. Meier, Deutsch. Arch.f. kl. Med., 1928, 161, 59.

74) Anrep n. Canna n, Journ. Physiol., 1923, 58, 244.

75) Long, Journ. Physiol., 1923, 58, 453.

76) Hayasaka u. Itak ura, Tohoku Journ. Exp. Med., 1931, 18, 175. 
$6,8 \% \mathrm{CO}_{2}$ das Absinken der Milchsäure in 7 von 9 Fällen nachgewiesen und dieses Absinken dahin erklürt, dass die durch Einatmung von kohlensäurereicher Luft bewirkte Azidosis die Adrenalinsekretion fördere und weil Adrenalin das Leberglykogen hinaus mobilisiert, zum Ersatz dieses Glykogenmangels die Milchsiure lebhaft resynthetisient werde und ferner dass das Natriumlaktat im Blutserum in Bestreben, die Azidosis zu nentralisieren, Na von sich abgebe und die hierbei freigewordene Milchsäure aus den Nieren ausgeschieden werde. Auch $J_{0} \circ{ }^{i \pi}$ konstatierte an Menschen durch die Einatmung eines Gasgemisches von $5 \% \mathrm{CO}_{2}+95 \% \mathrm{O}_{2}$ eine Abnahme der Milchsäure um 1-3 $\mathrm{mg} \%$, ebenso anch Gesell und seine Mitarbeiter $\left.{ }^{28}\right)$ konnten durch die Einführung von $11,4 \% \mathrm{CO}_{2}$ eine Verminderung der Milchsäure nachweisen. Auch in meinem Versuch habe ich zur Übereinstimmung mit Ergebnissen vieler Au. toren eine Abnahme der Milchsäure nachgewiesen.

Diese Milchsänreabnahme dürfte, wie Hayasaka u. Itaku ra ${ }^{76}$ behaupten, zum Teil durch beschleunigte Milchsäureresynthese infolge der durch Azidosis bewirkte Steigerung der Adrenalinsekretion sowie durch gesteigerte Ausscheidung der Milchsäure aus den Nieren bedingt sein. Andererseits wäre aber auch wie Adachi und ich ${ }^{(9)}$ schon mitgeteilt haben, die Möglichkeit denkbar, dass die hierbei entstandene Azidosis auf die Glykolyse in den Muskeln stark hemmend einwirkt und somit die Milchsänrebildung beeinträchtigt, was naturgemäss zur Abuahme der Blutmilchsäure führen muss.

Es unterliegt mehr keinem $Z$ weifel, dass die Erniedrigung des $\mathbf{O}_{2^{-}}$ Verbrauchs, welche bei der Einatmung von kohlensäurereicher Luft auftritt, durch die Azidosis herbeigeführt wird.

Gesell, Ir ruegel, Gorham und Berntha lis) beobachteten, dass die Zufuhr von $\mathrm{CO}_{2}$ die $\mathrm{O}_{2}$-Aufnahme verminderte, Hayasaka u. Itakura ${ }^{20}$ konstatierten an Menschen auch dasselbe. Dagegen wollen Oda und Y amadabo) keine dentliche Veränderung des $\mathrm{O}_{2}$-Verbrauchs nachgewiesen haben, anch Herx heimer und $\mathrm{K}_{\text {os }} \mathrm{t}^{21}$ heben hervor, dass hierbei keine erhebliche Steigerung des $O_{2}$. Verbranchs auftrete und $S \mathrm{ch}$ wi $\mathrm{gk}^{23}{ }^{23}$ fand bei der Einatmung von $8 \% \mathrm{CO}_{2}$ eine geringfügige $Z$ unahme, $\mathrm{K}$ roe t $\mathrm{z}^{81}$ konstatierte bei der Inhalation von $5 \% \mathrm{CO}_{2}$ den vermehrten $\mathrm{O}_{2}$-Verbrauch. Fischer-Wasels und Büngele $\left.\mathrm{r}^{2} \mathrm{i}\right)$ haben der Meinung Ausdruck gegeben, dass der bei der Einatmung von Kohlensäure erfolgende Veränderung des $\mathrm{O}_{2}$-Verbrauchs von der $\mathrm{O}_{\mathrm{n}}$-Konzentration in respiriertem Gas abhängig sei, und dass bei der Inhalation eines Gasgemisches von $5 \% \mathrm{CO}_{2}+95 \% \mathrm{O}_{2}$ die Vermehrung des $\mathrm{O}_{2}$.Verbrauchs sich grösser als bei der Einatmung von $5 \% \mathrm{CO}_{2}+$ Luft erweise.

Die derartige Verschiedenheit in den Ergebnissen mag wohl von verschiedentlichen Versuchsbedingungen hergerührt haben; die Azidosis in verschiedenen Geweben dürfte meines Erachtens von vornherein eine Verminderung des

77) Joos, Frankfurter Zeitschr. f. Pathol, 1930, 39, 403.

78) Gesell, Frueger, Gorham u. Bernthal, Americ. Journ. Physiol, 1030, 94, 402

79) Kodera u. Adachi, Tohokn Journ. Exp. Med., 1932, 20, 178.

80) Oda u. Yamada, Nippon Naika Gakkai Zasshi, 1930, 18, 225.

81) Kroetz, Jahreskurse ärztl. Fortbild, 1931, 22, 21. 
$\mathrm{O}_{\mathbf{g}}$-Verbrauchs bewirken, beim Umstand aber, wo durch die Dyspnoe infolge der Einatmung von Kohlensänre und durch andere Momente die körperliche Anstrengung benötigt wird, findet wahrscheinlich eine Vermehrung des $\mathrm{O}_{\mathrm{g}}$-Verbrauchs statt. Epping er ${ }^{17}$ n. a. haben experimentell den Beweis dafür geliefert, dass bei der Einatmung von $5 \% \mathrm{CO}_{\mathrm{a}}$ wegen der Überventilation unter Umständen eher die Alkalosis zustande kommt.

Es ist schon oben angeführt worden, dass Pulsverlangsamung und Steigerung der Atemzahl durch den zentralen, durch $\mathrm{CO}_{2}$ bedingten Reiz verursacht werden.

\section{Laktatinjektion unter der Inhalation von kohlensäurereicher Luft.}

Das zur Inhalation benutzte Gas war ein Gasgemisch von 8,0$12,9 \% \mathrm{CO}_{2}$ und 20,3-21,4\% $\mathrm{O}_{2}$. Das Ergebnis in 7 Versuchen ist aus Tab. 4 und Fig. 4 ersichtlich.

Der Gehalt an Blut- $\mathrm{CO}_{2}$ war vor Inhalation der kohlensäurereichen Luft bei 42,3-54,1, durchschnittlich zu 49,2 Vol. ó gelegen. 20 Minuten nach Eröffnung der Inhalation, nämlich unınittelbar vor Lak-

$$
\text { Tabelle } 4 .
$$

Versuch bei Laktatinjektion unter der Einatmung von kohlensäurereicher Luft.

\begin{tabular}{|c|c|c|c|c|c|c|c|c|}
\hline Nr. & 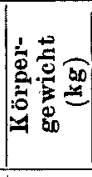 & $\begin{array}{c}\text { Zeit } \\
\text { (Min.) }\end{array}$ & 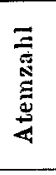 & 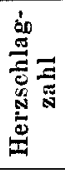 & 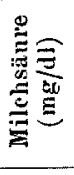 & 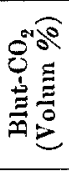 & 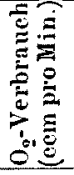 & Bemerkungen \\
\hline 1 & $\begin{array}{l}2,21 \\
(9)\end{array}$ & $\begin{array}{l}\text { Vor Inhalation } \\
\text { Vor Injektion } \\
5^{\prime} \text { nach } \\
15^{\prime} " \\
30^{\prime} " \\
60^{\prime} "\end{array}$ & & & $\begin{array}{l}19,3 \\
14,1 \\
54,0 \\
46,8 \\
33,0 \\
20,6\end{array}$ & $\begin{array}{l}50,1 \\
66,7 \\
72,3 \\
68,6 \\
68,6 \\
68,6\end{array}$ & $\begin{array}{l}15,5 \\
21,4 \\
21,3 \\
20,1 \\
18,6\end{array}$ & $\begin{array}{l}\text { Inhalationsluft: } \\
\mathrm{CO}, 9,9 \%, \mathrm{O}_{2} 20,3 \\
\% \text {. } \\
5,5 \text { ccm } 15 \% \mathrm{Natr} \text {. } \\
\text { lact. i. v. }\end{array}$ \\
\hline 2 & $\begin{array}{l}2,2 \\
(9)\end{array}$ & $\begin{array}{l}\text { Vor Inhalation } \\
\text { Vor Injektion } \\
5^{\prime} \text { nach } \\
15^{\prime} " \\
30^{\prime} " \\
60^{\prime} "\end{array}$ & & & $\begin{array}{l}20,3 \\
16,1 \\
45,0 \\
43,1 \\
34,1 \\
28,9\end{array}$ & $\begin{array}{l}50,3 \\
63,4 \\
70,8 \\
76,4 \\
74,6 \\
74,6\end{array}$ & $\begin{array}{l}15,2 \\
22,7 \\
23,2 \\
18,6 \\
17,0\end{array}$ & $\begin{array}{l}\text { Inhalationsluft: } \\
\mathrm{CO}_{2} 12,9 \%, \mathrm{O}_{2} 20,7 \\
\% . \\
5,5 \text { ccm } 15 \% \mathrm{Natr} \text {. } \\
\text { lact. i. v. }\end{array}$ \\
\hline 3 & $\begin{array}{l}1,81 \\
(q)\end{array}$ & $\begin{array}{l}\text { Vor Inhalation } \\
\text { Vor Injektion } \\
5^{\prime} \text { nach } \\
15^{\prime} " \\
30^{\prime} " \\
60^{\prime} "\end{array}$ & $\begin{array}{l}72 \\
86 \\
56 \\
68 \\
84 \\
84\end{array}$ & $\begin{array}{l}240 \\
204 \\
228 \\
240 \\
246 \\
224\end{array}$ & $\begin{array}{l}20,4 \\
16,1 \\
44,1 \\
40,5 \\
32,9 \\
23,9\end{array}$ & $\begin{array}{l}42,9 \\
67,3 \\
71,0 \\
71,0 \\
69,1 \\
69,1\end{array}$ & $\begin{array}{l}18,5 \\
28,1 \\
27,0 \\
26,9 \\
24,5\end{array}$ & $\begin{array}{l}\text { Inhalationsluft: } \\
\mathrm{CO}_{2} 8,5 \%, \mathrm{O}_{2} 20,9 \\
\% . \\
4,5 \mathrm{ccm} 15 \% \mathrm{Natr} . \\
\text { lact. i. v. }\end{array}$ \\
\hline
\end{tabular}




\begin{tabular}{|c|c|c|c|c|c|c|c|c|}
\hline $\mathrm{Nr}$. & 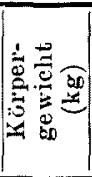 & $\begin{array}{c}\text { Zeit } \\
\text { (Min.) }\end{array}$ & 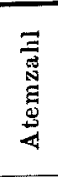 & 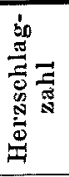 & 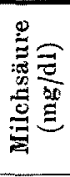 & 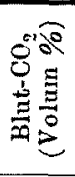 & 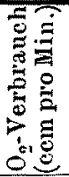 & Bemerkungen \\
\hline 4 & $\begin{array}{l}1,9 \\
(q)\end{array}$ & $\begin{array}{l}\text { Vor Inhalation } \\
\text { Vor Injektion } \\
5^{\prime} \text { nach } \\
15^{\prime} " \\
30^{\prime} " \\
60^{\prime} "\end{array}$ & $\begin{array}{r}70 \\
114 \\
114 \\
108 \\
108 \\
90\end{array}$ & $\begin{array}{l}222 \\
232 \\
264 \\
264 \\
276 \\
264\end{array}$ & $\begin{array}{l}21,2 \\
16,9 \\
48,2 \\
41,9 \\
34,1 \\
23,9\end{array}$ & $\begin{array}{l}54,0 \\
66,1 \\
66,1 \\
68,0 \\
70,8 \\
70,8\end{array}$ & $\begin{array}{l}21,4 \\
28,1 \\
28,9 \\
26,7 \\
18,5\end{array}$ & $\begin{array}{l}\text { Inhalationsluft: } \\
\mathrm{CO}_{2} 8,0 \%, 0_{2} 21,4 \\
\% . \\
4,75 \mathrm{ccm} 15 \% \mathrm{Natr} . \\
\text { lact. i. v. }\end{array}$ \\
\hline 5 & $\begin{array}{l}1,8 \\
\left(\begin{array}{l}q \\
q\end{array}\right)\end{array}$ & $\begin{array}{l}\text { Vor Inhalation } \\
\text { Vor Injektion } \\
5^{\prime} \text { nach } \\
1 \sigma^{\prime} " \\
30^{\prime} " \\
60^{\prime} "\end{array}$ & $\begin{array}{r}68 \\
92 \\
126 \\
120 \\
114 \\
114\end{array}$ & $\begin{array}{l}282 \\
204 \\
276 \\
288 \\
306 \\
300\end{array}$ & $\begin{array}{l}27,0 \\
18,0 \\
51,4 \\
38,7 \\
30,2 \\
23,1\end{array}$ & $\begin{array}{l}50,9 \\
57,5 \\
60,4 \\
62,2 \\
62,2 \\
61,3\end{array}$ & $\begin{array}{l}17,0 \\
21,8 \\
21,8 \\
20,2 \\
19,5\end{array}$ & $\begin{array}{l}\text { Inlialationsluft: } \\
\mathrm{CO}_{2} 8,0 \%, 0_{2} 20,5 \\
\% \text {. } \\
4,6 \mathrm{ccm} 15 \% \text { Natr. } \\
\text { lact. i. v. }\end{array}$ \\
\hline 6 & $\begin{array}{l}2,3 \\
(?)\end{array}$ & $\begin{array}{l}\text { Vor Inhalation } \\
\text { Vor Injektion } \\
5^{\prime} \text { nach } \\
15^{\prime} " \\
30^{\prime} " \\
60^{\prime} "\end{array}$ & $\begin{array}{r}90 \\
84 \\
132 \\
96 \\
84 \\
80\end{array}$ & $\begin{array}{l}210 \\
128 \\
200 \\
204 \\
216 \\
220\end{array}$ & $\begin{array}{l}29,3 \\
19,9 \\
44,4 \\
39,9 \\
33,4 \\
26,4\end{array}$ & $\begin{array}{l}42,3 \\
62,5 \\
51,5 \\
51,5 \\
53,3 \\
58,8\end{array}$ & $\begin{array}{l}21,2 \\
31,4 \\
30,9 \\
27,8 \\
26,9\end{array}$ & $\begin{array}{l}\text { Inhalationsluft: } \\
\mathrm{CO}_{2} 8,2 \%, \mathrm{O}_{2} 20,6 \\
\% \text {. } \\
5,8 \text { cem } 15 \% \text { Natr. } \\
\text { lact. i. v. }\end{array}$ \\
\hline 7 & $\begin{array}{l}2,2 \\
(\hat{\delta})\end{array}$ & $\begin{array}{l}\text { Vor Inhalation } \\
\text { Vor Injektion } \\
5^{\prime} \text { nach } \\
15^{\prime} ", \\
30^{\prime} " \\
60^{\prime} "\end{array}$ & $\begin{array}{l}72 \\
96 \\
96 \\
88 \\
84 \\
88\end{array}$ & $\begin{array}{l}204 \\
188 \\
234 \\
204 \\
222 \\
232\end{array}$ & $\begin{array}{l}30,2 \\
21,2 \\
60,4 \\
54,5 \\
43,7 \\
33,4\end{array}$ & $\begin{array}{l}54,1 \\
61,5 \\
62,4 \\
70,8 \\
72,7 \\
76,4\end{array}$ & $\begin{array}{l}18,4 \\
26,9 \\
21,9 \\
19,7 \\
26,4\end{array}$ & $\begin{array}{l}\text { Inhalationsluft: } \\
\mathrm{CO}_{2} 8,3 \%, \mathrm{O}_{2} 20,7 \\
\% \text {. } \\
5,5 \text { cem } 15 \% \mathrm{Natr} \text {. } \\
\text { lact. i. v. }\end{array}$ \\
\hline \multicolumn{2}{|c|}{ Mittelwert } & $\begin{array}{l}\text { Vor Inhalation } \\
\text { Vor Injektion } \\
5^{\prime} \text { nach } \\
15^{\prime} \text { " } \\
30^{\prime} " \\
60^{\prime} "\end{array}$ & $\begin{array}{r}74 \\
94 \\
105 \\
96 \\
95 \\
91\end{array}$ & $\begin{array}{l}232 \\
191 \\
240 \\
240 \\
253 \\
248\end{array}$ & $\begin{array}{l}24,0 \\
17,5 \\
49,6 \\
43,6 \\
34,5 \\
25,7\end{array}$ & $\begin{array}{l}49,2 \\
63,6 \\
64,9 \\
66,9 \\
67,3 \\
68,5\end{array}$ & $\begin{array}{l}18,2 \\
25,8 \\
25,0 \\
22,9 \\
21,6\end{array}$ & \\
\hline
\end{tabular}

tatinjektion betrug er 57,5-67,3 im Mittel 63,6 Vol.\%; ebenso wie in der vorigen Versuchsieihe nahm $\mathrm{CO}_{2}$ hier bedeutend zu. 5 Minuten nach Laktatinjektion war sie bei Versuch 4 konstant, bei Versuch 6 in geringem Masse vermindert. In anderen 5 Fällen zeigte die Blutkohlensäure entschieden mehr erhöhte Werte als dieselben, welche ohne Laktatzufuhr in demselben Zeitraum gemessen wurden. Diese Zunahme war jedoch ziemlich niedriger als bei der Inhalation der atmosphärischen Luft oder des Sauerstoffes.

Was den weiteren Verlauf anbelangt, nahm $\mathrm{CO}_{2}$ mitunter (Versuch 6) noch weiter in raschem Tempo zu, in den meisten Fällen aber behielt sie unter geringer Schwankung erhöhte Werte bei und kam nie auf die Werte vor Laktatzufuhr zurück. 
Fig. 4. Versuch bei Laktatinjektion unter der Inhalation von kohlensäurereicher Luft. (Versuch 3)

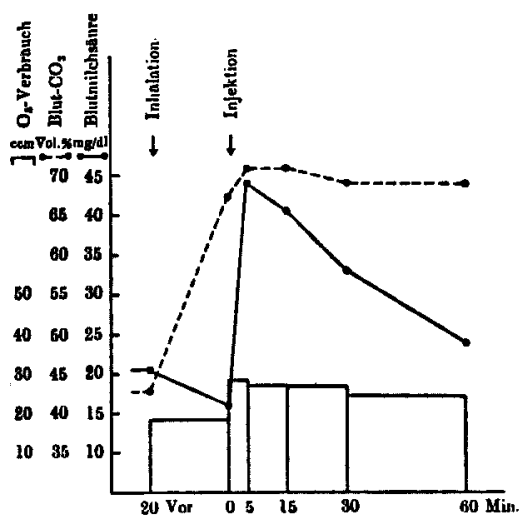

Die Blutmilchsäure hatte vor der Inhalation von Kohlensäure 19,3-30,2, im Mittel 24,0 $\mathrm{mg} / \mathrm{dl}$ betragen. Ii 20 Minuten nach Inhalation wurde ein Wert von 14,1-21,2, ein Mittelwert von $17,5 \mathrm{mg} / \mathrm{dl}$ ermittelt; in ganz derselben Weise wie in der vorigen Versuchsreihe nahm sie also ausnahmslos ab. In 5 Minuten nach Laktatzufuhr erreichte sie einen Wert von 44,1-60,4, einen Durchschnittswert von 49,6 $\mathrm{mg} / \mathrm{dl}$, ein Wert, der demjenigen Wert, welcher bei der Inhalation der atmosphärischen Luft oder des Sauerstoffes in derselben Zeitspanne ermittelt wurde, entweder nahezu gleichkommt oder etwas überlegen ist. Da aber der Blutmilchsäurespiegel, welcher unmittelbar vor Laktatzufuhr sich kundgibt, deutlich niedriger als bei der Einatmung der atmosphärischen Luft oder des Sauerstoffes ist, so sollte die Zunahme deutlich höher sein.

Was den hernach erfolgten Verlauf der Milchsäure anbelangt, nahm sie mit Ablauf der Zeit zwar ab, betrug dennoch nach 60 Minuten noch 20,6-33,4, im Mittel $25,7 \mathrm{mg} / \mathrm{dl}$; dieser Wert liegt von dem unmittelbar vor Laktatzufuhr ermittelten Wert ziemlich entfernt in allen Fällen.

Entgegen der Tatsache, dass die Milchsäure bei der Einatmung von Sauerstoff oder normaler Luft nach 30 oder 60 Minuten vollkommen wiederhergestellt war, war bei der Einatmung von kohlensäurereicher Luft die Resynthese der Milchsäure ausserordentlich behindert.

Der $\mathrm{O}_{\mathrm{z}}$-Verbrauch betrug bis zum Ablauf von 20 Minuten nach Inhalation 15,5-21,4 im Mittel 18,2 ccm pro Min. 5 Minuten nach Laktatzufuhr betrug er 21,4-31,4, durchschnittlich $25,8 \mathrm{ccm}$, er erwies sich also in allen Fällen erhöht, war aber niedriger als bei der Einatmung von gewöhnlicher Luft. Bezüglich des einschlägigen nachträglichen Verlaufes war der $\mathrm{O}_{2}$-Verbrauch nur bei Versuch 4 allein nach 60 Minuten zum vor Laktatzufuhr gefundenen Wert wiederhergestellt, während er in anderen 6 Versuchen allmählich abnahm, doch nach 60 Minuten einen mässig erhöhten Wert beibehielt. Kurzum, 
die durch Laktatzufuhr herbeigeführte Zunahme an $\mathrm{O}_{2}$-Verbrauch war zwar unbedeutend, hielt aber für längere Zeit an.

Von der durch Azidosis herbeizuführenden Verminderung des $\mathrm{O}_{\mathbf{a}^{-}}$ Verbrauchs war schon die Rede. Und bei $\mathrm{CO}_{2}$-Azidosis, wobei wie oben angeführt, die Milchsäureresynthese gestört wird und dadurch die durch Laktatinjektion herbeigeführte Vermehrung der Milchsäure äusserst verzögert auf den Ursprungswert zurückkommt, muss begreiflicherweise der vermehrte $\mathrm{O}_{2}$-Verbrauch länger als beim Kontrolltier anhalten.

\section{Versuche bei der Inhalation von sauerstoffarmer Luft.}

1. Einatmung von sauerstoffarmer Luft ohne die Laktatinjektion.

Hierbei hat das Tier eine Luft, die 8,7-10,5\% $\mathrm{O}_{2}$ enthielt, eingeatmet. Das Versuchsergebnis an 7 Tieren ist in Tab. 5 und Fig. 5 wiedergegeben.

Blut- $\mathrm{CO}_{2}$ betrug vor Inhalation von sauerstoffarmer Luft 45,765,7 , im Mittel 54,8 Vol. $\%$. In 20 Minuten nach Inhalation wurde

Tabelle 5.

Versuch unter der Einatmung von sauerstoffarmer Luft.

\begin{tabular}{|c|c|c|c|c|c|c|c|c|}
\hline $\mathrm{Nr}$. & 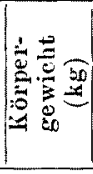 & $\begin{array}{l}\text { Zeit } \\
\text { (Min.) }\end{array}$ & ב⿱艹 & 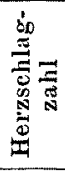 & 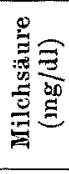 & 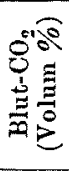 & 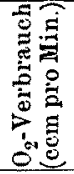 & Bemerkungen \\
\hline 1 & $\begin{array}{l}2,14 \\
(ㅇ ㅜ)\end{array}$ & $\begin{array}{l}\text { Vor Inhalation } \\
20^{\prime} \text { nach } \\
25^{\prime} \quad " \\
35^{\prime} " \\
50^{\prime} " \\
80^{\prime} "\end{array}$ & $\begin{array}{r}50 \\
140 \\
144 \\
152 \\
160 \\
144\end{array}$ & $\begin{array}{l}256 \\
224 \\
226 \\
234 \\
234 \\
236\end{array}$ & $\begin{array}{l}28,9 \\
27,0 \\
29,3 \\
36,5 \\
41,1 \\
32,0\end{array}$ & $\begin{array}{l}45,7 \\
41,1 \\
38,4 \\
35,7 \\
35,7 \\
36,6\end{array}$ & $\begin{array}{l}16,4 \\
18,9 \\
19,5 \\
22,0 \\
19,6\end{array}$ & $\begin{array}{c}\text { Inhalationsluft: } \\
\mathrm{O}_{2} 9,04 \% \\
\mathrm{CO}_{2} 0,05 \%\end{array}$ \\
\hline 2 & $\begin{array}{l}2,0 \\
(0)\end{array}$ & $\begin{array}{l}\text { Vor Inhalation } \\
20^{\prime} \text { nach } \\
25^{\prime} " \\
35^{\prime} " \\
50^{\prime} " \\
80^{\prime} "\end{array}$ & $\begin{array}{r}80 \\
132 \\
120 \\
119 \\
119 \\
120\end{array}$ & $\begin{array}{l}234 \\
188 \\
204 \\
204 \\
222 \\
234\end{array}$ & $\begin{array}{l}27,0 \\
23,6 \\
23,6 \\
29,3 \\
27,0 \\
25,3\end{array}$ & $\begin{array}{l}58,6 \\
35,7 \\
33,9 \\
26,5 \\
29,3 \\
29,3\end{array}$ & $\begin{array}{l}18,9 \\
17,6 \\
21,8 \\
22,0 \\
20,2\end{array}$ & $\begin{array}{c}\text { Inhalationsluft: } \\
\mathrm{O}_{2} 9,04 \% \\
\mathrm{CO}_{2} 0,04 \%\end{array}$ \\
\hline 3 & $\begin{array}{l}2.1 \\
(9)\end{array}$ & $\begin{array}{l}\text { Vor Inhalation } \\
20^{\prime} \text { nach } \\
25^{\prime} \quad " \\
35^{\prime} " \\
50^{\prime} " \\
80^{\prime} "\end{array}$ & $\begin{array}{r}60 \\
128 \\
144 \\
140 \\
152 \\
140\end{array}$ & $\begin{array}{l}276 \\
264 \\
224 \\
234 \\
234 \\
240\end{array}$ & $\begin{array}{l}20,4 \\
27,4 \\
30,7 \\
33,4 \\
30,7 \\
26,0\end{array}$ & $\begin{array}{l}45,7 \\
33,8 \\
32,9 \\
27,4 \\
21,0 \\
19,1\end{array}$ & $\begin{array}{l}18,0 \\
15,5 \\
14,4 \\
15,5 \\
13,1\end{array}$ & $\begin{array}{c}\text { Inhalationsluft: } \\
\mathrm{O}_{2} 8,70 \% \\
\mathrm{CO}_{2} 0,05 \% .\end{array}$ \\
\hline
\end{tabular}




\begin{tabular}{|c|c|c|c|c|c|c|c|c|}
\hline Nr. & 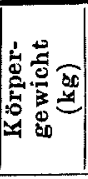 & $\begin{array}{l}\text { Zeit } \\
\text { (Min.) }\end{array}$ & 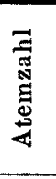 & 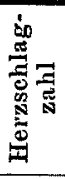 & 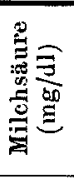 & 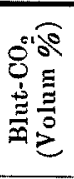 & 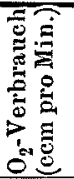 & Bemerkungen \\
\hline 4 & $\begin{array}{l}1,9 \\
(q)\end{array}$ & $\begin{array}{l}\text { Vor Inhalation } \\
20^{\prime} \text { nach } \\
25^{\prime} \quad " \\
35^{\prime} ", \\
50^{\prime} " \\
80^{\prime} "\end{array}$ & $\begin{array}{l}56 \\
82 \\
88 \\
92 \\
92 \\
88\end{array}$ & $\begin{array}{l}320 \\
270 \\
288 \\
288 \\
300 \\
304\end{array}$ & $\begin{array}{l}21,3 \\
27,0 \\
27,6 \\
29,7 \\
31,5 \\
36,5\end{array}$ & $\begin{array}{l}63,8 \\
51,1 \\
49,3 \\
45,6 \\
42,0 \\
35,6\end{array}$ & $\begin{array}{l}23,1 \\
20,0 \\
24,9 \\
24,4 \\
28,8\end{array}$ & $\begin{array}{c}\text { Inhalationsluft: } \\
\mathrm{O}_{2} 8,99 \% \\
\mathrm{CO}_{2} 0,02 \% .\end{array}$ \\
\hline 6 & $\begin{array}{l}2,05 \\
(9)\end{array}$ & $\begin{array}{l}\text { Vor Inhalation } \\
20^{\prime} \text { nach } \\
25^{\prime} " \\
35^{\prime} " \\
50^{\prime} " \\
80^{\prime} "\end{array}$ & $\begin{array}{r}42 \\
86 \\
86 \\
84 \\
80 \\
104\end{array}$ & $\begin{array}{l}292 \\
256 \\
260 \\
264 \\
264 \\
280\end{array}$ & $\begin{array}{l}25,7 \\
23,9 \\
29,6 \\
27,4 \\
34,1 \\
39,1\end{array}$ & $\begin{array}{l}65,7 \\
54,7 \\
46,5 \\
47,4 \\
48,3 \\
43,8\end{array}$ & $\begin{array}{l}17,1 \\
18,5 \\
17,3 \\
17,3 \\
16,0\end{array}$ & $\begin{array}{c}\text { Inhalationsluft: } \\
\mathrm{O}_{2} 9,65 \% \\
\mathrm{CO}_{2} 0,05 \% .\end{array}$ \\
\hline 6 & $\begin{array}{l}1,75 \\
(ㅇ ㅜ)\end{array}$ & $\begin{array}{l}\text { Vor Inhalation } \\
\mathbf{2 0 ^ { \prime }} \text { nach } \\
\mathbf{2 5 ^ { \prime }} " \\
\mathbf{3 5} " \\
\mathbf{5 0 ^ { \prime }} " \\
80^{\prime} "\end{array}$ & $\begin{array}{r}64 \\
120 \\
120 \\
120 \\
128 \\
128\end{array}$ & $\begin{array}{l}286 \\
276 \\
268 \\
280 \\
280 \\
280\end{array}$ & $\begin{array}{l}25,7 \\
27,0 \\
28,9 \\
30,9 \\
31,5 \\
29,6\end{array}$ & $\begin{array}{l}55,9 \\
43,3 \\
38,7 \\
43,3 \\
43,3 \\
37,8\end{array}$ & $\begin{array}{l}19,6 \\
20,5 \\
18,1 \\
17,6 \\
18,5\end{array}$ & $\begin{array}{c}\text { Inhalationsluft: } \\
\mathrm{O}_{2} 10,52 \% \\
\mathrm{CO}_{2} 0,01 \% .\end{array}$ \\
\hline 7 & $\begin{array}{l}2,0 \\
(q)\end{array}$ & $\begin{array}{l}\text { Vor Inhalation } \\
20^{\prime} \text { nach } \\
25^{\prime} " \\
35^{\prime} " \\
50^{\prime} " \\
80^{\prime} "\end{array}$ & $\begin{array}{r}46 \\
100 \\
104 \\
88 \\
96 \\
100\end{array}$ & $\begin{array}{l}266 \\
242 \\
240 \\
246 \\
240 \\
246\end{array}$ & $\begin{array}{l}25,7 \\
21,9 \\
23,8 \\
28,3 \\
28,3 \\
27,0\end{array}$ & $\begin{array}{l}47,9 \\
43,3 \\
39,6 \\
35,0 \\
36,9 \\
36,9\end{array}$ & $\begin{array}{l}16,7 \\
21,5 \\
20,7 \\
19,6 \\
21,3\end{array}$ & $\begin{array}{c}\text { Inhalationsluft: } \\
\mathrm{O}_{2} 9,90 \% \\
\mathrm{CO}_{2} 0,02 \%\end{array}$ \\
\hline \multicolumn{2}{|c|}{ Mittelwert } & $\begin{array}{l}\text { Vor Inhalation } \\
20^{\prime} \text { nach } \\
25^{\prime} " \\
35^{\prime} " \\
50^{\prime} " \\
80^{\prime} "\end{array}$ & $\begin{array}{r}57 \\
113 \\
115 \\
114 \\
118 \\
118\end{array}$ & $\begin{array}{l}276 \\
246 \\
244 \\
250 \\
253 \\
260\end{array}$ & $\begin{array}{l}25,0 \\
25,4 \\
27,6 \\
30,8 \\
32,0 \\
30,8\end{array}$ & $\begin{array}{l}54,8 \\
43,3 \\
39,9 \\
37,3 \\
36,6 \\
34,2\end{array}$ & $\begin{array}{l}18,5 \\
18,9 \\
19,5 \\
19,8 \\
19,6\end{array}$ & \\
\hline
\end{tabular}

Fig. 5. Versuch unter der Inhalation von sauerstoffarmer Luft. (Versuch 1)

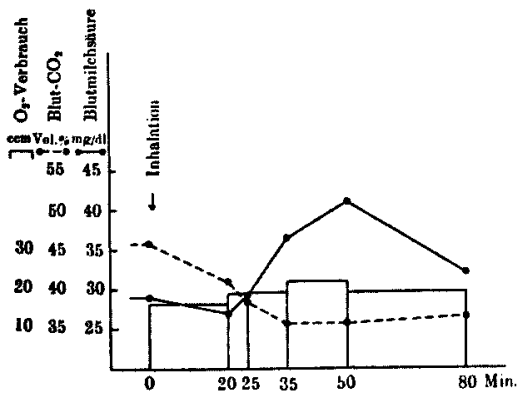

sie zu 33,8-54,7, durchschnittlich zu $43,3 \%$ gefunden, hier trat also ausnahmslos eine deutliche Abnahme ein. Was das nachher erfolgte Schicksal von Blut- $\mathrm{CO}_{\mathrm{s}}$ anbetrifft, nahm sie bei Versuch 1,2 und 7 bis zum Ablauf von 35 Minuten fortdauernd $a b$, neigte sich hernach aber zu geringfügiger Zunahme. Während sie bei Versuch 5 und 6 in 20 Minuten das Minimum er- 
reichte und nachher mehr oder weniger zunahm, war sie bei Versuch 3 und 4 hingegen bis zum Ablauf von 80 Minuten kontinuierlich vermindert.

Die Blutmilchsäure war vor Inhalation von sauerstoffarmer Luft bei 20,4-28,9, im Mittel bei 20,5 mg/dl gelegen. In 4 Fällen (Versuch 1, 2, 5 und 7) nahm sie 20 oder 25 Minuten nach Einatmung von diesem Gase ein wenig ab und hernach $z u$, in anderen 3 Fällen nahm sie schon vom Anfang an zu. In 2 Fällen (Versuch 4 und 5) stieg sie bis zum Ablauf von 80 Minuten fortlaufend an, während sie in anderen 5 Fällen in 35 bzw. 00 Minuten ihren Gipfelpunkt erreichte und darauf mehr oder minder zur Abnahme geneigt war.

Der $\mathrm{O}_{2}$-Verbrauch betrug innerhalb von 20 Minuten nach erfolgter Inhalation von sauer'stoffarmer Luft 16,4-23,1, in Durchschnitt $18,5 \mathrm{ccm}$ pro Min. Dieser Betrag war im Vergleich zu demselben bei der Einatmung von normaler Luft oder Sauerstoff offenbar vermindert. $\mathrm{Der} \mathrm{O}_{2}$-Verbrauch verlief nachher unter unerheblichen Schwankungen, diese gestalteten sich je nach den einzelnen Fällen verschieden und standen auch mit Veränderungen der Milchsäure keineswegs im Einklang.

Die Atemzahl wurde durch die Inhalation dieses Gases auf das Doppelte vermehrt und erhielt danach unter geringer. Schwankung bis zum Abschluss des Versuchs erhöhte Zahl. Die Pulszahl nahm 20 Minuten nach Inhalation gewissermassen ab, nachher allmählich zu und war geneigt auf die frühere Zahl wiederzukehren.

Schon in der Einleitung ist darauf hingewiesen worden, dass wenn der $\mathrm{O}_{2}$-Gehalt bis unter $14 \%$ reduziert ist, die Ventilationsgrösse zunimmt, wodurch die $\mathrm{CO}_{2}$-Spannung in den Lungenalveolen abnimmt und somit auch das Blut der Alkalosis anheimfällt. Die deutliche Abnahme an Blut- $\mathrm{CO}_{2}$ in meinem Versuch wäre dahin zu erklären, dass durch die hierbei eintretende Hyperventilation die $\mathrm{CO}_{3}$-Abgabe in übermässiger Weise erfolgt und dass zu einer Zeit, wo nach dem bestimmten Zeitablauf die Blutmilchsäure zunimmt, zum Neutralisieren dieser nicht flüchtigen Säure das Reservealkali ausgenutzt wird, was Blut- $\mathrm{CO}_{2}$ erneut zur Verminderung bringt.

Über die Beziehung $\mathrm{z}$ wischen den $\mathrm{O}_{2}$-Mangeln in der Atemluft und der Milchsäure ist in den letzten Jahren von vielen Forschern studiert worden. Als Hinweis darauf sei nachfolgend erwähnt.

Laque u $\mathrm{r}^{82}$ fand beim Versuch im Höhenklima, dass in der $2700 \mathrm{~m}$ Höhe die Milchsäure nur mässig zunimmt und in über $4500 \mathrm{~m}$ Höhe nicht mehr an-

82) Laqueur, Zeitschr. f. Biol,, 1919, 70, 99. 
steigt. Barcrof $\left.\mathrm{t}^{8 i}\right)$ konstatierte bei einer Höhe von 10,000 feet keine Steigerung der Milchsäure, in einer Höhe ron 15,000 feet aber eine deutliche Zunahme. Macleod ${ }^{30}$ berichtete darüber, dass bei akuter Anoxämie inmerhalb ron 10 Dinuten eine deutliche Znnahme der Milchsäure zustandekomme, Mc Ginty und $\mathrm{Ges}$ e $1^{8-1}$ beobachteten, dass die Milchsăurezunahme bei Asphyxie vor dem Ablauf vou 10 Jinuten nicht in Erscheinung tritt. Von Jervell ${ }^{85}$ ) ist mitge. teilt worden, dass bei Inhalation vou 10,20 und $100 \% \mathrm{O}_{2}$ keine $\ddot{\text { Änderung der }}$ Milchsäure venösen Blutes, aber erst bei Inhalation von $7,5 \%$ eine deutliche $\mathrm{Zu}$ nahme derselben sich erkenuen liesse. Ferner haben manche Autoren, wie Gesell, Krueger, Gorham u. Bernthal, ${ }^{78}$ Calabresi u. Schwarz ${ }^{8(i)}$ die Milchsäuresteigerung bein $\mathrm{O}_{2}$-Mangel nachgewiesen.

In meinem Versuch hat es sich herausgestellt, dass bei der Einatmung vor $8,7-10,5^{\circ} ; \mathrm{O}_{2}$ die Blutmilchsäure in 4 von 7 Fällen nach 20 Minuten zwar in geringem Masse abnimmt, nach 30 Minuten aber. auffallend zunimmt, während sie in anderen 3 Fällen vom Anfang an vermehrt gefunden wird. Nach Angaben von Macleod u. Hoover, ${ }^{8 i}$ Evans, ${ }^{85}$ Anrep u. Cannan, ${ }^{\text {(4) }}$ Eggleton u. Evans ${ }^{89}$ u.a. soll die Blutmilchsäure bei gesteigerter Alkalität des Blutes zunehmen.

Die Milchsäurezunalhme bei Inhalation von sauerstoffarmer Luft dürfte, wie von mir und $\mathrm{Sugimot0^{90 }}$ referiert worden ist, auf die durch Alkalosis bedingte Steigerung der Glykolyse und weiter noch auf den Umstand, dass infolge der unzulänglichen $\mathrm{O}_{2}$-Versorgung auf Gewebe, die durch den $\mathrm{O}_{2}$-Mangel im Blut und die herabgesetzte Reduktionsgeschwindigkeit des Hämoglobins hervorgerufen worden ist, die Oxydation übermässig gebildeter Milchsäure beeinträchtigt wird, zurückzuführen sein. Dies geht nämlich aus der $\mathrm{O}_{2}$-Aufnahme, die deutlich kleiner als normal geworden ist, ohne weiteres hervor.

Ửber die Veränderungen des $\mathrm{O}_{2}-$ Verbrauchs hat $\mathrm{Y}$ os o mi y $\mathrm{a}^{(4)}$ in hiesiger Klinik im Versuch des progressiven $\mathrm{O}_{2}$-Mangels bei Kaninchen festgestellt, dass der $\mathrm{O}_{2}$-Verbrauch anfänglich entweder konstant oder vermehrt ist, aber dann, wenn er unter $10 \%$ heruntersinkt, erheblich abnimmt. Ebenso auch $\mathrm{Okada} \mathrm{a}^{25}$ in hiesiger Klinik hat bei der Inhalation von $8 \% \mathrm{O}_{2}$ den verminderten $\mathrm{O}_{2}$-Verbrauch nachgewiesen. Dass infolge des verminderten $\mathrm{O}_{2}$-Verbrauchs die Milchsäure-

83) Barcroft, The respiratory function of the blood, Cambridge 1925, Part I, 76.

84) McGinty u. Gesell, Americ. Journ. Physiol, 1925, 75, 70.

85) Jervell, Acta Medica Scandinavica, Suppl. xxiv, Chapter 6.

86) Calabresi u. Schwarz, Riforma med, 1930, 1111, ref. in Kongresszentral. f. ges. inn. Med., 1931, 60, 663 .

87) Macleod n. Ho over, Americ. Journ. Physiol., 1917, 42, 460.

88) Evans, Journ. Physiol., 1922, 56, 146.

89) Eggleton u. Evans, Journ. Physiol., 1930, 70, 261.

90) Kodera n. Sugimoto, wird bald in dieser Zeitschr. publiziert. 
zunahme sich geltend macht, ist wohl annehmbar; die Tatsache aber, dass diese Milchsäurezunahme dann, wenn sie eine bestimmte Höhe erreicht hat, nicht mehr so manifest in den Vordergrund tritt, wird wenigstens zum Teil darauf beruhen, dass die Milchsäure durch Bestreben der Niere und anderer Organe ausserhalb des Körpers ausgeschieden wird.

Über die gesteigerte Michsäureausscheidung im Harn bei erhöhter Blutalkalität ist von $\mathrm{Macleod^{91) }}$ und $\mathrm{Macleod}$ sowie $\mathrm{Knapp}{ }^{92)}$ erwiesen worden.

\section{Laktatinjektion unter der Einatmung von sauerstoffarmer Luft.}

Hierbei wurde in 5 Kaninchen unter Einatmenlassen der 8,679,76\% $\mathrm{O}_{2}$ enthaltenden Luft der Versuch ausgeführt. Das Ergebnis ist in Tab. 6 u. Fig. 6 zusammengestellt.

Blut- $\mathrm{CO}_{2}$ nahm durch die Einatmung von sauerstoffarmer Luft in eben derselben Weise wie in der vorangegangenen Versuchsreihe be-

Tabelle 6.

Versuch bei Laktatinjektion unter der Einatmung von sauerstoffarmer Luft.

\begin{tabular}{|c|c|c|c|c|c|c|c|c|}
\hline $\mathrm{Nr}$. & 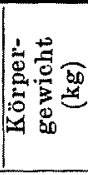 & $\begin{array}{c}\text { Zeit } \\
\text { (Min.) }\end{array}$ & 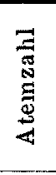 & 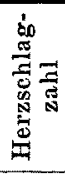 & 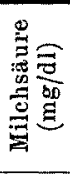 & 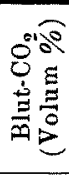 & 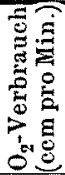 & Bemerkungen \\
\hline 1 & $\begin{array}{l}2,5 \\
(q)\end{array}$ & $\begin{array}{l}\text { Vor Inhalation } \\
\text { Vor Injektion } \\
5^{\prime} \text { nach } \\
15^{\prime} " \\
30^{\prime} " \\
60^{\prime} "\end{array}$ & $\begin{array}{r}84 \\
122 \\
124 \\
116 \\
116 \\
112\end{array}$ & $\begin{array}{l}248 \\
228 \\
254 \\
256 \\
262 \\
252\end{array}$ & $\begin{array}{l}23,6 \\
20,0 \\
60,1 \\
57,8 \\
54,4 \\
49,8\end{array}$ & $\begin{array}{l}44,3 \\
34,1 \\
38,6 \\
35,8 \\
33,1 \\
34,1\end{array}$ & $\begin{array}{l}16,1 \\
21,8 \\
23,3 \\
19,7 \\
19,5\end{array}$ & $\begin{array}{l}\text { Inhalationsluft: } \\
\mathrm{O}_{2} 9,55 \%, \mathrm{CO}_{2} 0,01 \\
\% \% \\
6.25 \text { ccm } 15 \% \mathrm{Natr} \text {. } \\
\text { lact. i. v. }\end{array}$ \\
\hline 2 & $\begin{array}{l}2,23 \\
\text { (우) }\end{array}$ & $\begin{array}{l}\text { Vor Inhalation } \\
\text { Vor Injektion } \\
55^{\prime} \text { nach } \\
15^{\prime} " \\
30^{\prime} " \\
60^{\prime} ",\end{array}$ & $\begin{array}{r}56 \\
166 \\
180 \\
176 \\
152 \\
150\end{array}$ & $\begin{array}{l}282 \\
256 \\
282 \\
280 \\
280 \\
280\end{array}$ & $\begin{array}{l}27,0 \\
28,8 \\
48,6 \\
43,2 \\
35,1 \\
32,4\end{array}$ & $\begin{array}{l}58,8 \\
49,6 \\
53,3 \\
34,0 \\
34,0 \\
34,0\end{array}$ & $\begin{array}{l}17,6 \\
22,8 \\
21,5 \\
22,8 \\
24,0\end{array}$ & $\begin{array}{l}\text { Inhalationsluft: } \\
\mathrm{O}_{2} 9,76 \%, \mathrm{CO}_{2} 0,02 \\
\% \% \text {. } \\
5,5 \text { ccm } 15 \% \text { Natr. } \\
\text { lact. } i . \mathrm{v} \text {. }\end{array}$ \\
\hline 3 & $\begin{array}{l}2,15 \\
(9)\end{array}$ & $\begin{array}{l}\text { Vor Inhalation } \\
\text { Vor Injektion } \\
5^{\prime} \text { nach } \\
15^{\prime} " \\
30^{\prime} " \\
60^{\prime} "\end{array}$ & $\begin{array}{r}76 \\
102 \\
116 \\
104 \\
108 \\
104\end{array}$ & $\begin{array}{l}240 \\
236 \\
256 \\
224 \\
226 \\
234\end{array}$ & $\begin{array}{l}22,5 \\
25,7 \\
45,0 \\
43,7 \\
37,6 \\
30,9\end{array}$ & $\begin{array}{l}51,1 \\
38,3 \\
38,3 \\
35,7 \\
35,7 \\
35,7\end{array}$ & $\begin{array}{l}18,4 \\
20,4 \\
20,2 \\
19,6 \\
19,6\end{array}$ & $\begin{array}{l}\text { Inhalationsluft: } \\
\mathrm{O}_{2} 8,67 \%, \mathrm{CO}_{2} 0,00 \\
\overline{0} \% \text {. } \\
5,3 \text { cem } 15 \% \mathrm{Natr} . \\
\text { lact. i. } v \text {. }\end{array}$ \\
\hline
\end{tabular}

91) M a cleod, A meric. Journ. Physiol., 1918, 45, 539.

92) Macleod u. Knapp, Americ. Journ. Physiol., 1918, 47, 189. 


\begin{tabular}{|c|c|c|c|c|c|c|c|c|}
\hline Nr. & 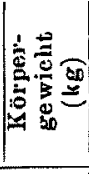 & $\begin{array}{c}\text { Zeit } \\
\text { (Min) }\end{array}$ & 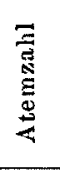 & $\frac{\dot{s}}{\stackrel{s}{ \pm}}=$ & $\begin{array}{l}\stackrel{0}{0}= \\
= \\
=\end{array}$ & 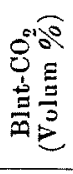 & 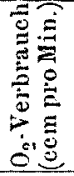 & Bemerkungen \\
\hline 4 & $\begin{array}{l}2,0 \\
(9)\end{array}$ & $\begin{array}{l}\text { Vor Inhalation } \\
\text { Vor Injektion } \\
5^{\prime} \text { nach } \\
15^{\prime} " \\
30^{\prime} " \\
60^{\prime} "\end{array}$ & $\begin{array}{r}60 \\
88 \\
92 \\
92 \\
88 \\
100\end{array}$ & $\begin{array}{l}222 \\
204 \\
240 \\
256 \\
248 \\
248\end{array}$ & $\begin{array}{l}22,5 \\
20,7 \\
69,9 \\
66,3 \\
58,8 \\
51,2\end{array}$ & $\begin{array}{l}51,4 \\
44,3 \\
46,6 \\
44,3 \\
43,2 \\
43,2\end{array}$ & $\begin{array}{l}16,4 \\
21,6 \\
216 \\
19,8 \\
20,9\end{array}$ & $\begin{array}{l}\text { Inhalationsluft: } \\
\mathrm{O}_{2} 9,01 \%, \mathrm{CO}_{2} 0,01 \\
\% \text {. } \\
5,0 \mathrm{ccm} 15 \% \mathrm{Natr} . \\
\text { lact. i. v. }\end{array}$ \\
\hline 5 & $\begin{array}{l}2.17 \\
(\delta)\end{array}$ & $\begin{array}{l}\text { Vor Inhalation } \\
\text { Vor Injektion } \\
5^{\prime} \text { nach } \\
15^{\prime} ", \\
30^{\prime} ", \\
60^{\prime} \quad "\end{array}$ & $\begin{array}{r}48 \\
126 \\
122 \\
122 \\
122 \\
122\end{array}$ & \begin{tabular}{|l}
300 \\
284 \\
296 \\
294 \\
300 \\
304
\end{tabular} & $\begin{array}{l}21,7 \\
250 \\
42,1 \\
39,4 \\
33,4 \\
27,0\end{array}$ & $\begin{array}{l}46,7 \\
35,0 \\
37,3 \\
38,2 \\
37,3 \\
35,0\end{array}$ & $\begin{array}{l}17,6 \\
25,1 \\
23,7 \\
23,6 \\
24,6\end{array}$ & $\begin{array}{l}\text { Inhalationsluft: } \\
0,8,95 \%, \mathrm{CO}_{2} 0,04 \\
0 \% \text {. } \\
5,5 \text { ecm } 15 \% \mathrm{Natr} \text {. } \\
\text { lact. i. v. }\end{array}$ \\
\hline \multicolumn{2}{|c|}{ Mittelwert } & $\begin{array}{l}\text { Vor Inhalation } \\
\text { Vor Injektion } \\
5^{\prime} \text { nach } \\
15^{\prime}, " \\
30^{\prime} \text { " } \\
60^{\prime} \text { " }\end{array}$ & $\begin{array}{r}65 \\
121 \\
127 \\
122 \\
117 \\
118\end{array}$ & $\begin{array}{l}258 \\
242 \\
266 \\
262 \\
263 \\
264\end{array}$ & $\begin{array}{l}23,5 \\
24,0 \\
53,1 \\
50,1 \\
43,9 \\
25,3\end{array}$ & $\begin{array}{l}50,6 \\
40,3 \\
42,8 \\
37,6 \\
36,7 \\
36,4\end{array}$ & $\begin{array}{l}17,2 \\
22,3 \\
22,1 \\
21,1 \\
21,7\end{array}$ & \\
\hline
\end{tabular}

Fig. 6. Versuch bei Laktatinjektion unter der Inhalation von sauerstoffarmer Luft. (Versuch 5)

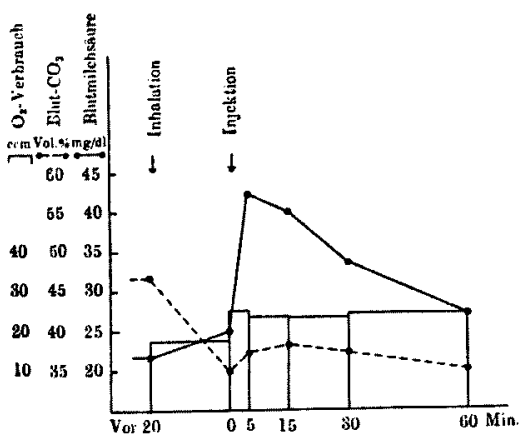

trächtlich ab. Im Ablauf von 5 Minuten nach Laktatzufuhr war sie in Versuch 3 konstant, in übrigen 4 Fällen um 2,3-4,5 Vol. $\%$ vermehrt. Diese Vermehrung erwies sich aber als entschieden kleiner als dieselbe bei der Einatmung der normalen oder sauerstoffreichen Luft. Bezüglich des nachträglich erfolgten Verlaufes ist zu erwähnen, dass bei Versuch 5 allein der $\mathrm{O}_{2}$-Verbrauch nach weiteren

15 Minuten unerheblich zunahm, in allen anderen Fällen allmählich immer mehr abnalım.

Die Blutmilchsäure nahm nach $\frac{1}{3}$ stündiger Einatmung von einer derartigen Luft in 2 (Versuch $1 \mathrm{u} .4$ ) von 5 Versuchen etwas ab, während sie in ubbrigen 3 Fällen zunahm, der Mittelwert wies nur in geringem Masse eine Zunahme auf. 5 Minuten nach Laktatzufuhr aber stieg sie auf 42,1-69,9, im Mittel 53,1 mg/dl hinauf, diese Erhöhung ist ausscrordentlich grecsser in Vergicich zu Werten bei Inlalation 
von atıosphärischer bzw. kohlensäurercicher Luft in demselben Zeitraum. Sie nahm nachher z war allmählich ab, die Geschwindigkeit der Abnahıne war überaus trägc, so dass die Milchsäure selbst nach 60 Minuten noch $27,0-51,2$, im Mittel $38,2 \mathrm{mg} /$ dl betrug. Dieser Wert liegt bei weitem entfernt von demjenigen Wert, der vor Laktatzufuhr ermittelt wurde, ebensowenig erreichte er den Mittelwert von 30,8 $\mathrm{mg} / \mathrm{dl}$, der ohne Laktatzufuhr in derselben Zeitspanne gemessen wurde. Hieraus kann gefolger't werden, dass die Oxydation und Resynthese der Milchsäure ausserordentlich gestört war:

Der $\mathrm{O}_{-}$-Verbrauch betrug binnen 20 Minuten nach Einatmung von sauerstoffarmer Luft 16,1-18,4, im Mittel 17,2 ccm pro Min.; er nahın also ebenso wie in der vorigen Versuchsreihe des $\mathrm{O}_{2}$-Mangels auffallend ab. Innerhalb von 5 Minuten nach Laktatzufuhr zeigte er einen Wert von 20,4-25,1, also einen Mittelwert von $22,3 \mathrm{ccm}$; er nahm in allen Fällen zu; diese Zunahme ist weitaus kleiner als die bei der Einatmung von normaler oder sauerstoffreicher Luft.

In weiter nachfolgenden 10 Minuten war er um einen geringen Grad vermehrt bei Versuch 1, bei Versuch 4 konstant, bei anderen 3 Fällen spärlich vermindert; es wurde ein Mittelwert von $22,1 \mathrm{ccm}$ ermittelt, was eine iusserst geringe Zunahme war. In weiteren $15 \mathrm{Mi}$ nuten war der $\mathrm{O}_{2}$-Verbrauch nur bei Versuch 2 allein geringfügig vermehrt, bei anderen 4 Fällen spärlich vermindert. In den letzten 30 Minuten nahm er bei 3 Fällen (Versuch 2, 4 und 5) zu, blieb bei Versuch 1 und 3 fast konstant, so dass er im Mittelwert vielmehr eine geringe Zunahme aufwics.

Obige Ergebnisse können nämlich dahin zusammengefasst werden, dass die Vermehrung des $\mathrm{O}_{-}$-Verbrauchs durch Laktatzufuhr unbedeutend ist und die andauernde Vermehrung beim $\mathrm{O}_{2}$-Mangel am auffallendsten zutage tritt.

Aus verzögerter Erholung des Milchsäurespiegels und geringfügiger Zunalıme an $\mathrm{O}_{3}$-Vorbrauch sowie aus dem längsten Andauern derselben kann man darauf schliessen, dass beim $\mathrm{O}_{2}$-Mangel überhaupt die Oxydation un: Resynthese der Milchsïure überaus gestört wird.

Was Veränderungen der A temzahl durch Laktatzufuhr anbelangr, nahm sie je nach den Fällen in sehr verschiedenem Masse zu, bei Versuch 5 nahm sie eher spärlich ab. Die Pulszahl nahm vor Laktatzufuhr transitorisch ab, nach Zufuhr vermehrte sie sich und erhielt diese vermehrte $Z$ ahl fortlaufend.

Dass die gestörte Resynthese des zugeführten milchsauren Natriums wie oben erwähnt durch die Beeinträchtigung der $\mathrm{O}_{2}$-Versorgung auf Gewebe hervorgerufen wird, bedarf keiner Erörterung und weil diese Beeintıächtigung der $\mathrm{O}_{2}$-Versorgung eine Herabsetzung 
der Oxydationsprozesse zur Folge hat, reduziert sich auch die Zunahme des $\mathrm{O}_{2}$-Verbrauchs auf das kleinste Mass.

Da aber die durch die Oxydation entstandene $\mathrm{CO}_{2}$ mit dem aus milchsaurem Natrium freigewordenen $\mathrm{Na}$ in eine Verbindung eingeht und nunmehr als die gebundene Kohlensäure die hierbei herrschende Hypokapnie mildert, stellt sich immerhin eine Vermehrung von $\mathrm{CO}_{2}$ im Blut ein, jedoch infolge der sehr bescheidenen $\mathrm{Oxy}$ dation erfolgt die $\mathrm{CO}_{2}$-Zunahme verständlicherweise nur in beschränktem Masse. Da auch andererseits die Milchsäureresynthese infolge beeinträchtigter Oxydation sehr gestört wird und deshalb die zugeführte Milchsäure längere Zeit verweilt, hält die Vermehrung des $\mathrm{O}_{2}$-Verbrauchs und der Blut-CO $\mathrm{CO}_{2}$ aturgemäss in wenn auch geringerem Masse, doch länger an.

\section{Zusammenfassung.}

Um mich über den Einfluss der Einatmung von sauerstoffreicher bzw. -armer Luft oder von kohlensäurereicher Luft auf den Milchsäurestoff wechsel zu orientieren, habe ich, indem ich obige Gase Kaninchen einatmen liess, beiihnen Veränderungen von Blut- $\mathrm{CO}_{2}$, Blutmilchsäure und $\mathrm{O}_{2}$-Verbrauch verfolgt, weiterhin habe ich Kaninchen im Laufe der Inhalation derartiger Gase $2,5 \mathrm{ccm} 15 \%$ iger Natriumlaktatlösung pro $\mathrm{kg}$ intravenös injiziert und alsdann die Oxydation und Resynthese des zugeführten Natriumlaktates mit denselben bei Einatmung von freier Luft vergleichend unter'sucht.

1. Bei der Einatmung von freier Luft tritt im direkten Anscliluss an die Laktatzufuhr eine Zunahme an $\mathrm{Blut}-\mathrm{CO}_{2}$, Blutmilchsäure und $\mathrm{O}_{2}$-Verbruch auf, und von dieser Zunahme erholt sich die Blut-CO. nach 60 Minuten, der Blutmilchsäurespiegel nach 30-60 Ninuten, der $\mathrm{O}_{2}$-Verbrauch nach 60 Minuten in vollkommener Weise.

2. Bei der Einatmung von reinem Sauerstoff treten Veränderungen von Blut-CO, Blutmilchsäure und $\mathrm{O}_{2}$-Verbrauch nicht so manifest in Erscheinung. Nach Zufuhr des Natriumlaktates werden die Werte obiger Dinge für gewisse Zeitabstände vermelnt gefunden, und was die Vermehrung im einzelnen anbelangt, erweist sich die Vermehrung des $\mathrm{O}_{2}$-Verbrauchs allein mehr oder minder stärker als die bei Inhalation der Luft von normaler Zusammensetzung, während andere Komponenten beinahe in gleichem Ausmasse zunelımen. Was die Erholung von derartigen vermehrten Werten anbetrifft, kommt die Blut$\mathrm{CO}_{2}$ in 30-60 Minuten völlig auf den Urspr'ungswert zurück, auch der Blutmilchsäurespiegel sowie der $\mathrm{O}_{2}-\mathrm{Verbrauch}$ werden in der Mehrzahl der Fälle in 30-60 Minuten vollkommen zum fiüheren Wert wiederhergestellt. Hinsichtlich der Oxydation und Resynthese der Milchsäure 
lässt sich also kein deutlicher Unterschied zwischen der Einatmung von normaler Luft und derselben von reinem Sauerstoff nachweisen.

3. Bei der Einatmung von Luft, die $7-10 \% \mathrm{CO}_{2}$ enthält, wird die Blut- $\mathrm{CO}_{2}$ anfänglich rasch und dann langsam vermehrt. Es wird nämlich durch die Einatmung dieses kohlensäurereichen Gases die $\mathrm{CO}_{-}$Azidosis bewerkstelligt. Die Blutmilchsäure nimmt im Laufe der 80 Minuten langen Einatmung obigen Gases ausnahmslos allmählich ab, der $\mathrm{O}_{2}$-Verbrauch nimmt auch etwas mehr als bei der Einatmung von atmosphärischer Luft $a b$.

Nach Laktatinjektion, die während der Einatmung von kohlensäurereicher Luft erfolgte, erhöht sich Blut- $\mathrm{CO}_{2}$ in stärkerem Masse als ohne die Injektion, aber in schwächerem als bei der Einatmung von atmosphärischer Luft.

Der Blutmilchsäurespiegel wird höher als bei Kontrolltieren gesteigert, erweist sich als beträchtlich höher im Vergleich zum Wert, der bei cler Einatmung von derselben Gasart ohne Laktatzufuhr in demselben Zeitraum ermittelt wird.

Der $\mathrm{O}_{2}$-Verbrauch nimmt in geringerem Masse als bei Kontrolle $\mathrm{zu}$, hält dafür länger an. Das kommt nämlich daher, dass die Oxydation in Geweben durch die $\mathrm{CO}_{-2}$-Azidosis gestört, und demgemäss die Milchsäureresynthese sehr verzögert wird.

4. Bei der Einatmung von $10 \%$ oder noch weniger Sauerstoff enthaltender Luft nimmt die Blut- $\mathrm{CO}_{2}$ am Anfang mit raschem Schritt und danach unter geringer: Sch wankung anhaltend mehr oder weniger ab.

Die Blutmilchsäure lässt bis zum Ablauf von 20 Minuten nach erfolgter Einatmung von obiger Luft keine merkliche Veränderung erkennen, nimmt aber darauf allmählich immer mehr zu und erreicht in 35-50 Min. das Maximum. Der $\mathrm{O}_{2}$-Verbrauch nimmt in vorwiegender Mehrzahl der Fälle ab.

Auch bei der unter Einatmung von sauerstoffarmer Luft erfolgten Laktatzufuhr nimmt Blut- $\mathrm{CO}_{2}$ in den meisten Fällen nach 5 Minuten in geringem Nasse zu und dann allmählich ab. Der Blutmilchsäurespiegel nimmt 5 Minuten nach Laktatzufuhr ausgesprochen zu, nachher sehr langsam ab, und zwar derart, dass er selbst nach 60 Min. noch einen ziemlich erhöhten Wert aufweist.

Der $\mathrm{O}_{2}$-Verbrauch nimmt in kleinerem Masse als bei der Einatmung von atmosphärischer Luft zu und behält doch diesen mässig erhöhten Wert bis zum Abschluss des Versuchs fast unverändert bei. Hieraus geht nämlich hervor, dass die $\mathrm{O}_{\mathrm{g}}$-Versorgung auf Gewebe durch die Einatmung von sauerstoffar'mer Luft beeinträchtigt und infalgedessen die Oxydation und Resynthese der Milchsäure auffallend gestbirt wird. 